\title{
Interpreting the Helioseismic and Magnetic Imager (HMI) Multi-Height Velocity Measurements
}

\author{
Kaori Nagashima ${ }^{1} \cdot$ Björn Löptien ${ }^{2}$. \\ Laurent Gizon ${ }^{1,2}$. Aaron C. Birch ${ }^{1}$. \\ Robert Cameron $^{1}$. Sebastien Couvidat ${ }^{3}$. \\ Sanja Danilovic ${ }^{1}$. Bernhard Fleck ${ }^{4}$. \\ Robert Stein ${ }^{5}$
}

(C) Springer $\bullet \bullet \bullet \bullet$

\begin{abstract}
The Solar Dynamics Observatory/Helioseismic and Magnetic Imager (SDO/HMI) filtergrams, taken at six wavelengths around the Fe I $6173.3 \AA$ line, contain information about the line-of-sight velocity over a range of heights in the solar atmosphere. Multi-height velocity inferences from these observations can be exploited to study wave motions and energy transport in the atmosphere. Using realistic convection simulation datasets provided by the STAGGER and MURaM codes, we generate synthetic filtergrams and explore several methods for estimating Dopplergrams. We investigate at which height each synthetic Dopplergram correlates most strongly with the vertical velocity in the model atmospheres. On the basis of the investigation, we propose two Dopplergrams other than the standard HMI-algorithm Dopplergram produced from HMI filtergrams: a linecenter Dopplergram and an average-wing Dopplergram. These two Dopplergrams correlate most strongly with vertical velocities at the heights of $30-40 \mathrm{~km}$ above (line-center) and 30-40 km below (average-wing) the effective height of the HMIalgorithm Dopplergram. Therefore, we can obtain velocity information from two layers separated by about a half of a scale height in the atmosphere, at best. The phase shifts between these multi-height Dopplergrams from observational data as well as those from the simulated data are also consistent with the heightdifference estimates in the frequency range above the photospheric acoustic cutoff frequency.
\end{abstract}

Keywords: Velocity Fields, Photosphere; Oscillations, Solar; Helioseismology, Observations

\footnotetext{
1 Max-Planck-Institut für Sonnensystemforschung, Justus-von-Liebig-Weg 3, 37077 Göttingen, Germany email: nagashima@mps.mpg.de

2 Institut für Astrophysik, Georg-August-Universität

Göttingen, 37077 Göttingen, Germany

3 Stanford University, Stanford, CA 94305, USA

4 ESA Science Operations Department, c/o NASA/GSFC,

Greenbelt, MD 20771, USA

5 Michigan State University, East Lansing, MI 48824, USA
} 


\section{Introduction}

In recent helioseismology studies multi-height velocity and intensity information have been used in addition to standard photospheric Dopplergrams. MitraKraev, Kosovichev, and Sekii (2008) investigated the phase shift between photosphericand chromospheric-intensity datasets obtained by the Hinode satellite. They reported large phase differences along the $p$-mode ridges and no phase difference on the $f$-mode ridge. Nagashima et al. (2009) inferred chromospheric downflows by interpreting multi-height observations. More recently, Howe et al. (2012) examined the phase differences between several observables originating from various layers obtained by the Helioseismic and Magnetic Imager (HMI: Scherrer et al. 2012) and the Atmospheric Imaging Assembly (AIA: Lemen et al., 2012) onboard the Solar Dynamics Observatory (SDO: Pesnell, Thompson, and Chamberlin, 2012). Rajaguru et al. (2012) exploited multi-height HMI and AIA data to study power enhancement around active regions at various heights in the atmosphere. They used not only the standard HMI-algorithm Dopplergrams but also Doppler information derived from the line wing (this is similar to what we define as the "far-wing" Dopplergram in Section 2.3.

Multi-height information is, however, useful not only for helioseismology studies but also for many other research purposes, for example, the study of energy transport in the solar atmosphere (e.g. Jefferies et al., 2006, Straus et al., 2008, Straus et al. 2009, Bello González et al., 2010, Kneer and Bello González, 2011). If we could obtain multi-height velocity information from SDO/HMI datasets, we would have huge datasets available compared with other current instruments; HMI obtains full-disk datasets without significant interruptions.

Here we show that we can obtain multi-height velocity information from SDO/HMI observations; we use realistic numerical convection simulations to characterize these multi-height Dopplergrams. In Section 2 we introduce HMI observables as well as the simulation datasets, and define several types of Dopplergrams. In Section 3 we investigate the contribution heights of the Doppler velocities using realistic convection simulations. On the basis of these contribution height estimates and availability of the observables we choose a pair of Doppler velocities as rather robust multi-height velocity datasets; this is summarized in Section 3.1. We measure the phase difference derived from the HMI observation datasets as well as simulated datasets in Section 4 . Finally, a brief summary is given in Section 5 .

\section{Making Multi-Height Dopplergrams from SDO/HMI Datasets}

\subsection{HMI Observables}

$\mathrm{SDO} / \mathrm{HMI}$ takes full-disk images in the Fe I absorption line at $\lambda_{\mathrm{lc}}=6173.3433$ $\AA$ (at rest). Every 45 seconds 12 filtergrams are taken at left- and right-circular polarizations at six wavelength positions around the line center: $+172 \mathrm{~m} \AA\left(\lambda_{0}\right)$, $+103.2 \mathrm{~m} \AA\left(\lambda_{1}\right),+34.4 \mathrm{~m} \AA\left(\lambda_{2}\right),-34.4 \mathrm{~m} \AA\left(\lambda_{3}\right),-103.2 \mathrm{~m} \AA\left(\lambda_{4}\right)$, and $-172 \mathrm{~m} \AA$ $\left(\lambda_{5}\right)$. Example filter profiles and the reference line profile used in the standard 


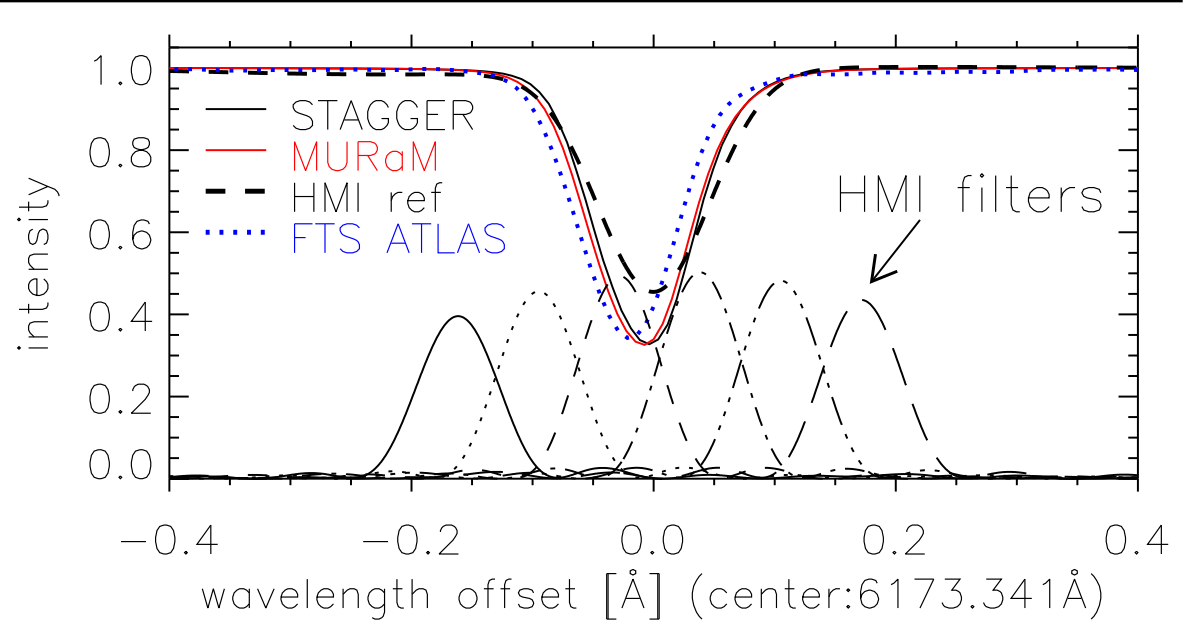

Figure 1. Profile of the Fe I absorption line at $6173.3 \AA$. The HMI reference line profile, NSO FTS atlas profile, synthetic line profiles of STAGGER and MURaM data averaged over each FOV, and HMI filter profiles are shown. The central wavelength positions of the filter are +172 $\mathrm{m} \AA\left(\lambda_{0}\right.$, long-dashed $),+103.2 \mathrm{~m} \AA\left(\lambda_{1}\right.$, dash-triple-dotted $),+34.4 \mathrm{~m} \AA\left(\lambda_{2}\right.$, dash-dotted $),-34.4$ $\mathrm{m} \AA$ ( $\lambda_{3}$, short-dashed $),-103.2 \mathrm{~m} \AA\left(\lambda_{4}\right.$, dotted $)$, and $-172 \mathrm{~m} \AA\left(\lambda_{5}\right.$, solid $)$ from right to left.

HMI data processing are shown in Figure 1 (see Couvidat et al., 2012 for details). The HMI reference line profile is a simple combination of Voigt and Gaussian functions which are calibrated for the purpose of obtaining Dopplergrams (Schou et al. 2014). Figure 1 also shows the National Solar Observatory (NSO) Fourier Transform Spectrometer (FTS) Atlas spectra (Wallace, Hinkle, and Livingston, 1998), the reference line profile used in the HMI data processing and also the synthesized line profiles (Section 2.2.3). The pixel size is 0.505 arcsec, or $370 \mathrm{~km}$ on the Sun at the disk center. In this article we consider only the data obtained by the front camera, which is the camera for the line-of-sight observables.

The standard Dopplergram products of the HMI pipeline (hereafter, HMIalgorithm Dopplergrams) are made from these sets of filtergrams (Couvidat et al. 2012), and the formation height of the HMI-algorithm Dopplergram is around $100 \mathrm{~km}$ above $\tau_{5000 \AA}=1$ (Fleck, Couvidat, and Straus, 2011), while the line-core formation height is estimated to be around 200-300 km (e.g. Norton et al. 2006).

In this study, we show that by combining the HMI filtergrams at six wavelengths in several ways we can derive multi-height velocity information in the solar atmosphere. Note that we always take the sum of the intensities at leftand right-circular polarizations at every wavelength, and hereafter we use only these total intensities $\left[I_{i}(i=0,1, \ldots, 5)\right.$ at $\left.\lambda_{i}\right]$. In the HMI pipeline, on the other hand, HMI-algorithm Doppler velocities are computed separately from the left- and right-circular polarization intensities, and then the two Doppler velocities are averaged. Since the data processing is non-linear, this might cause small differences from our approach. In this study, however, we limit ourselves to quiet-Sun data. Thus the two approaches should produce similar results. 


\subsection{Realistic 3D Convection Simulation Datasets}

To estimate the contribution heights of "multi-height" Dopplergrams, we use datasets from two different simulation codes: STAGGER (Stein et al. 2009 a; 2009b:Stein, 2012) and Max-Planck-Institut für Sonnensystemforschung/University of Chicago RAdiative MHD (MURaM: Vögler et al., 2005) code. The average profiles of the solar atmosphere models in these simulations are shown in Figure 2 as well as a standard solar model (Model S: Christensen-Dalsgaard et al., 1996) and the VAL atmosphere (Vernazza, Avrett, and Loeser, 1981) for comparison. Since we also compare our results with the results from COnservative COde for the COmputation of COmpressible COnvection in a BOx of $\mathrm{L}$ Dimensions with I=2,3 (CO ${ }^{5}$ BOLD: Freytag, Steffen, and Dorch, 2002, Wedemeyer et al., 2004) at several points, we show these profiles as well. The height $[z]$ is defined relative to the layer where $\tau_{5000} \AA=1$. The differences in the mean structure in the three simulations relative to Model S are within $10 \%$ at the surface. However, at $400 \mathrm{~km}$ above the surface the pressure and the density differences between the models are up to $30 \%$ and $25 \%$ while the temperature difference is $5 \%$. A detailed comparison of these models is given by Beeck et al. (2012).

\subsubsection{Simulated Data}

2.2.1.1. STAGGER Data We use a $3 \mathrm{D}$ convection simulation provided by the STAGGER code. The simulation domain is $96 \mathrm{Mm}$ wide and extends vertically from the temperature minimum down to a depth of $20 \mathrm{Mm}$. There is only weak photospheric magnetic field; the average unsigned vertical field is $3 \mathrm{G}$, the average unsigned horizontal field is $5 \mathrm{G}$, and the maximum field is $2.2 \mathrm{kG}$ at the surface. The horizontal resolution is $48 \mathrm{~km}$, and the vertical resolution varies from $12 \mathrm{~km}$ near the surface to $80 \mathrm{~km}$ at large depths. From this domain we use only a subregion for the radiative-transfer calculations. The subregion is $48 \mathrm{Mm} \times 48 \mathrm{Mm}$ wide and extends from the $550 \mathrm{~km}$ above to $450 \mathrm{~km}$ below the surface with $\tau_{5000} \AA=1$. This $\tau_{5000} \AA$ is defined by the average atmospheric profiles shown in Figure 2

2.2.1.2. MURaM Data We also use convection-simulation results provided by the MURaM code with non-gray radiative transport using four opacity bins (Vögler, Bruls, and Schüssler, 2004). The region we use is $9 \mathrm{Mm}$ wide in the horizontal directions and covers $3 \mathrm{Mm}$ in the vertical direction (from $1.98 \mathrm{Mm}$ below the surface up to $1 \mathrm{Mm}$ above the $\tau_{5000 \AA}=1$ level) with $17.6 \mathrm{~km}$ horizontal resolution and $10 \mathrm{~km}$ vertical resolution. This simulation is non-magnetic.

\subsubsection{Spatial Resolution}

The original pixel sizes of STAGGER and MURaM simulation datacubes are 48 $\mathrm{km}$ and $18 \mathrm{~km}$, respectively, and are much smaller than the HMI pixel size (370 $\mathrm{km}$ on the Sun at the disk center), or the HMI diffraction limit (1.83 HMI pixels). As discussed by Fleck, Couvidat, and Straus (2011), the spatial resolution may affect the analyses. Here we use a theoretical point spread function (PSF) to 


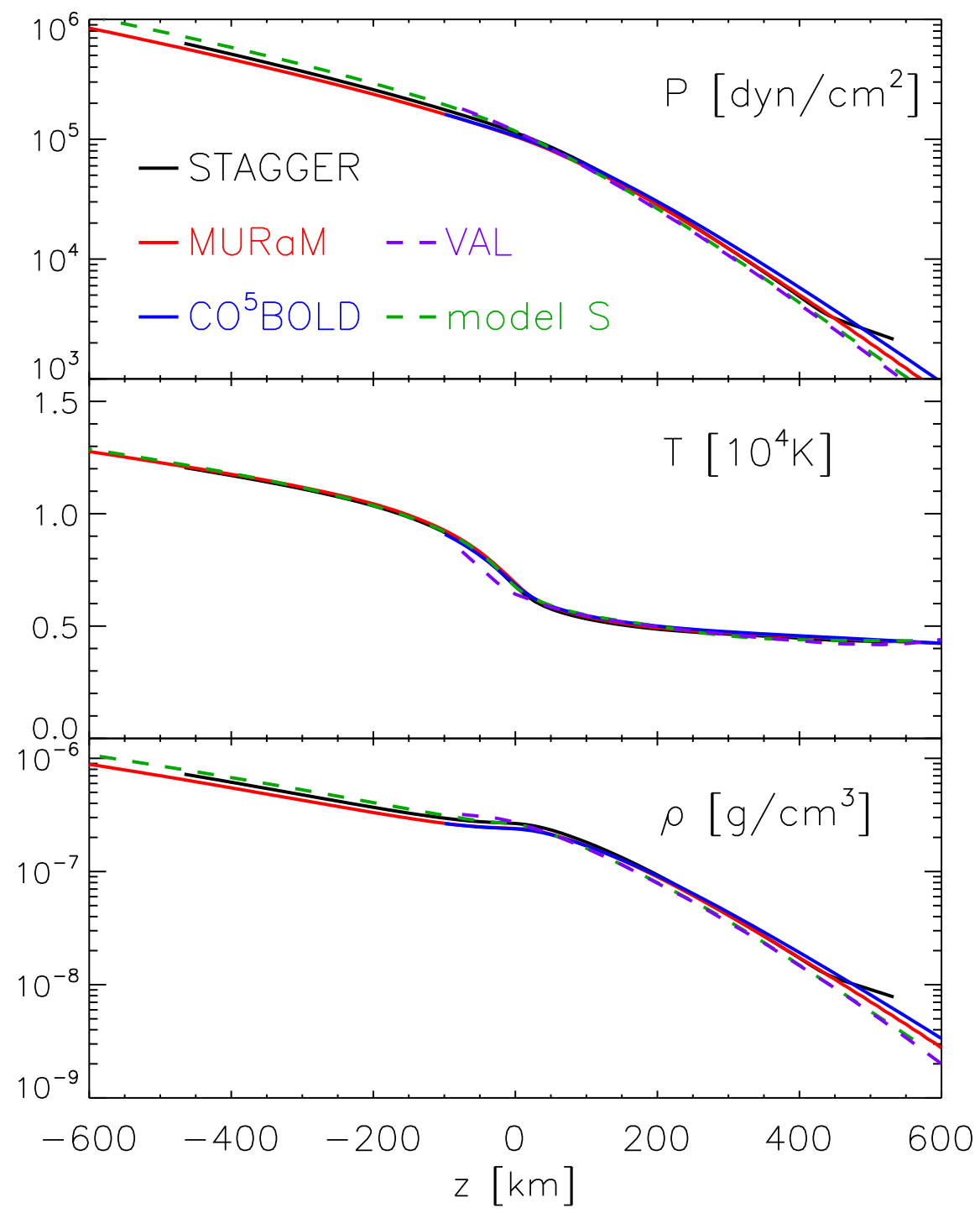

Figure 2. Average atmospheric profiles of STAGGER (black solid), MURaM (red solid), and $\mathrm{CO}^{5} \mathrm{BOLD}$ (blue solid) simulations as well as Model S (green dashed) and VAL (purple dashed). The zero point of the height $z$ is defined as the height where $\tau_{5000 \AA}=1$.

synthesize HMI observables from the simulation datasets. The theoretical PSF $[P]$ for an optical system with aperture diameter $[D]$ and focal length $[f]$ at wavelength $[\lambda]$ is given by

$$
P\left(r^{\prime}\right)=\left[\frac{2 J_{1}\left(r^{\prime}\right)}{r^{\prime}}\right]^{2}
$$


where $J_{1}$ is the first-order Bessel function of the first kind, $r^{\prime}=\pi D r /(\lambda f)$ is the normalized radius, and $r$ is the radial distance from the optical axis on the focal plane (e.g. Stix, 2002). The first zero of $P$ is at $r^{\prime}=1.22 \pi$, and the diffraction limit for HMI is defined here as $r_{\text {dif.limit }}=\lambda f / D=21.9 \mu \mathrm{m}$, where $\lambda=\lambda_{\text {lc }}$ and the effective focal ratio $f / D=35.42$ (Schou et al., 2012$)$. The HMI CCD pixel size is $12 \mu \mathrm{m}$, so the diffraction limit corresponds to $1.83 \mathrm{HMI}$ pixels.

Because of the limited field of view of the MURaM data, we apply this PSF to the STAGGER data only. If we apply the PSF to the MURaM data, we have only $\approx 10^{2}$ data points in the field of view.

\subsubsection{Synthetic Spectra and Filtergrams}

Using the simulated datasets, we calculate the line profile for the Fe I absorption line at $6173.3 \AA$ using the Stokes-Profiles-INversion-O-Routines (SPINOR) code (Frutiger et al., 2000 ). The atomic parameters that we use to synthesize the line profiles are summarized in Table 1. The abundance is from Bellot Rubio and Borrero (2002). Since this line is formed relatively deep in the photosphere, the assumption of LTE for the line synthesis calculations seems justified for the particular objectives of this article (cf., e.g., Bello González et al., 2009; Fleck, Couvidat, and Straus, 2011). The average line profiles obtained from STAGGER and MURaM data are shown in Figure 1. Figure 3 shows a set of snapshots of synthetic filtergrams created from synthetic spectra convolved with the HMI filter profiles, and Figure 4 shows the filtergrams obtained from the STAGGER datasets after the PSF was applied. For comparison, Figure 4 also shows the HMI filtergrams taken at 07:29:15 UT on 23 January 2011 near the disk center. At the time of the observation, the SDO line-of-sight motion toward the Sun was 288.25 $\mathrm{m} \mathrm{s}^{-1}$ and this is subtracted when we calculate the Doppler velocities from these filtergrams (shown in Figure6 in Section 2.3). The filtergrams created from the simulation datasets (upper panels of Figure 4) look qualitatively similar to the observations (lower panels of Figure 4), although the fact that the structure sizes in observation datasets seem slightly bigger than those in the synthesized datasets may indicate that the real PSF for HMI is slightly worse than the purely theoretical one we used here.

Table 1. Atomic parameters for the line synthesis.

\begin{tabular}{cccccc}
\hline \multirow{2}{*}{ Wavelength } & Atomic mass & Fe abundance & log gf & 1st & 2nd \\
\hline $6173.341 \AA$ & 55.8500 & 7.43 & -2.880 & $2.222 \mathrm{eV}$ & $7.9024 \mathrm{eV}$ \\
\hline
\end{tabular}

\subsection{Several Types of Doppler Velocity Measurements}

We calculate several types of velocities from the synthetic line spectra and the filtergrams. Although only six filtergrams are available from the HMI observations, here we derive these quantities from full spectra as well (5 and 6 in the 

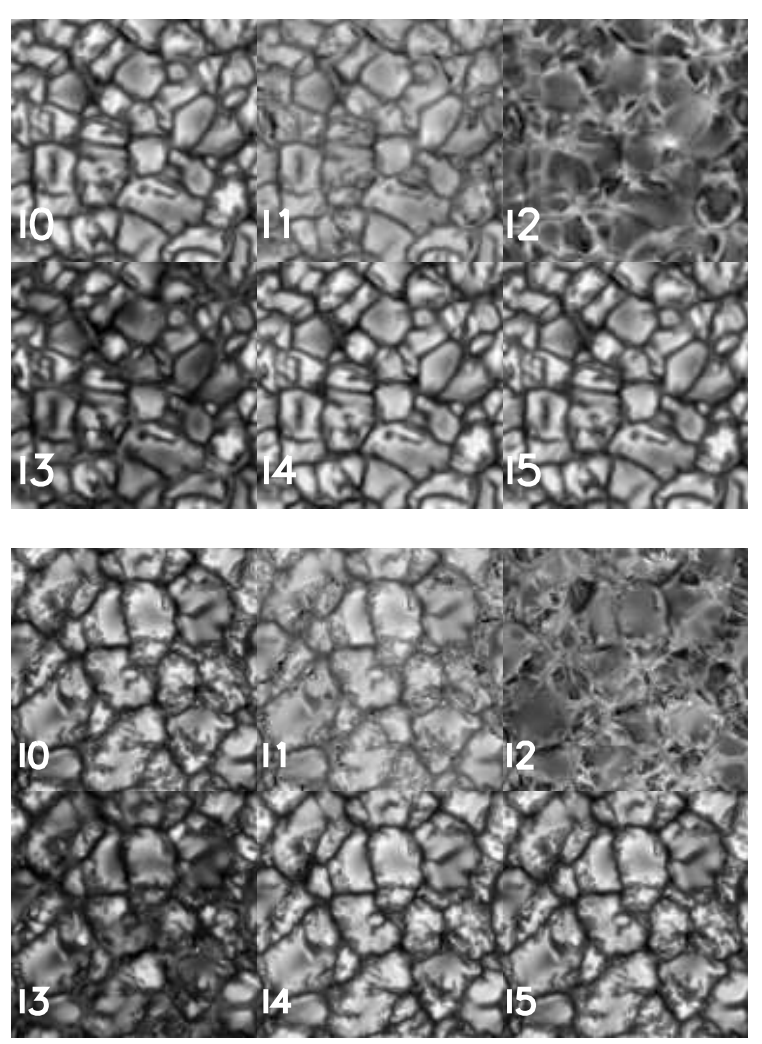

Figure 3. Sample synthetic filtergrams obtained from the STAGGER datasets (top) and MURaM datasets (bottom). The labels $I_{i}(i=0,1, \ldots, 5)$ indicate filtergrams with the filter centered at $\lambda_{i}$. The area of the STAGGER maps is $10 \mathrm{Mm}$ square, which is about one-fifth of the original field of view. The area of the MURaM maps is $9 \mathrm{Mm}$ square, the full original field of view. With these original resolutions, the granular pattern is clearly seen in any wavelength, although the contrast depends on the wavelength.

list below) for the synthetic data analyses. We compute the intensities at 200 points in wavelength from $-0.75 \AA$ to $+0.75 \AA$ around the line center using SPINOR. Although there are still some tiny sidelobes in the HMI filters outside this range (see Couvidat et al., 2012 for details), they do not matter for the analyses performed here. In the calculation of the absorption-line profile, we do not include the turbulent velocity in the simulated atmosphere. Therefore, the line profile for each pixel shows a narrower and steeper profile compared with the line profile averaged over the entire FOV (shown in Figure 1).

\subsubsection{Doppler Velocity 1: Core, Wing, Far-Wing, and Average-Wing Doppler Velocities}

These Doppler velocities are derived from the Doppler signal made of pairs of filtergrams,

$$
D_{\mathrm{br}}=\left(I_{\mathrm{b}}-I_{\mathrm{r}}\right) /\left(I_{\mathrm{b}}+I_{\mathrm{r}}\right),
$$



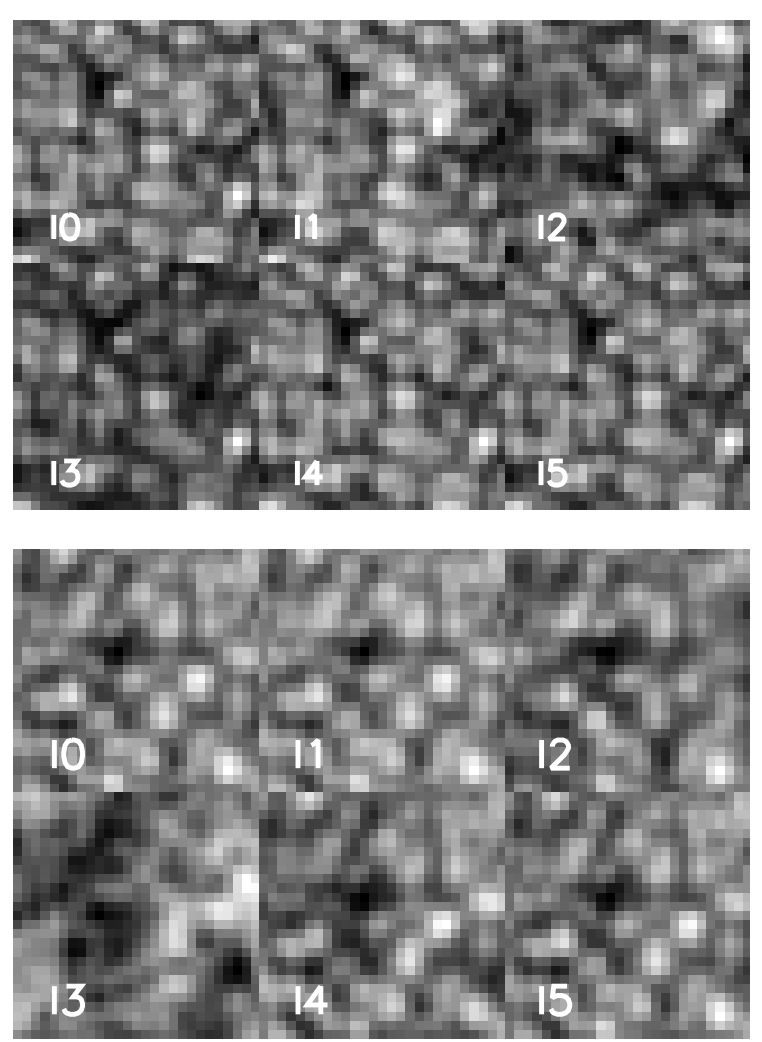

Figure 4. Sample filtergrams obtained from STAGGER datasets with the resolution reduced by an approximate HMI PSF (top) and example HMI observations (bottom). The labels $I_{i}$ $(i=0,1, \ldots, 5)$ indicate filtergrams with the filter centered at $\lambda_{i}$. The area is $10 \mathrm{Mm}$ square and the field of view of the top panel is identical to the STAGGER maps (top panels) of Figure 3 The top and bottom panels look reasonably similar. With this resolution, each granular cell structure is marginally resolved.

where $I_{\mathrm{b}}$ and $I_{\mathrm{r}}$ are the intensities of the blue and red sides of each pair. We define the core pair as $\left(I_{\mathrm{r}}, I_{\mathrm{b}}\right)=\left(I_{2}, I_{3}\right)$, the wing pair as $\left(I_{\mathrm{r}}, I_{\mathrm{b}}\right)=\left(I_{1}, I_{4}\right)$, the far-wing pair as $\left(I_{\mathrm{r}}, I_{\mathrm{b}}\right)=\left(I_{0}, I_{5}\right)$, and the average-wing pair as $\left(I_{\mathrm{r}}, I_{\mathrm{b}}\right)=\left(\left[I_{0}+\right.\right.$ $\left.\left.I_{1}\right] / 2,\left[I_{4}+I_{5}\right] / 2\right)$. Conversion of the Doppler signal into velocities is discussed in Appendix A.

\subsubsection{Doppler Velocity 2: Center of Gravity (Six Points)}

The Doppler velocity of the center of gravity $(\operatorname{cog})$ of the line is derived from

$$
v_{\operatorname{cog}}=c\left(\frac{\sum_{i=0}^{5} \lambda_{i}\left\{I_{i}-I_{\max }\right\}}{\lambda_{\mathrm{lc}} \sum_{i=0}^{5}\left\{I_{i}-I_{\max }\right\}}-1\right),
$$

where $\lambda_{\mathrm{l} c}$ is the line-center wavelength, $c$ is the speed of light, $\lambda_{i}$ is the wavelength where the intensity $I_{i}$ is measured, and $I_{\max }$ is the maximum of $I_{i}(i=0,1, \ldots, 5)$. 


\subsubsection{Dopler Velocity 3: Line Center (Three Points)}

The Doppler velocity of the line center can be estimated from the three wavelength points around the minimum-intensity wavelength. We calculate the parabol through the three points and use the apex of the parabola as an estimate of the line-center shift.

\subsubsection{Doppler Velocity 4: Simplified HMI-Algorithm Dopplergrams}

We calculate simplified HMI-algorithm Doppler velocities [ $v_{\text {HMI1 }}$ and $v_{\text {HMI2 }}$ ] based on the method used for HMI pipeline products (Couvidat et al. 2012 ). The procedure here is identical to that of Fleck, Couvidat, and Straus (2011). First we calculate the phase of the first and second Fourier coefficients $\left[\phi_{\mathrm{F} 1}\right.$ and $\left.\phi_{\mathrm{F} 2}\right]$ of the line profile (made of six filtergrams, namely, $I_{i}$ for $\left.i=0,1, \ldots, 5\right)$. Then we obtain the velocities as $v_{\mathrm{HMI} 1}=\alpha \phi_{\mathrm{F} 1} /(2 \pi)$ and $v_{\mathrm{HMI} 2}=\alpha \phi_{\mathrm{F} 2} /(4 \pi)$, where $\alpha=c / \lambda_{\mathrm{lc}}(68.8[\mathrm{~m} \AA] \times 6)$ (see Couvidat et al., 2012 for details). Note that the widely-used HMI-algorithm Dopplergram provided by the HMI pipeline is the first one $\left[v_{\mathrm{HMI}}\right]$, and the HMI-algorithm Dopplergrams mentioned in this article are $v_{\text {HMI1 }}$ unless otherwise noted.

\subsubsection{Doppler Velocity 5: Center of Gravity (Full)}

The Doppler velocity of the center of gravity of the full line profiles is calculated by

$$
v_{\mathrm{cog}, \mathrm{f}}=c\left(\frac{\int_{\lambda_{\mathrm{m}}}^{\lambda_{\mathrm{M}}} \mathrm{d} \lambda \lambda\left(I(\lambda)-I_{\mathrm{c}}\right)}{\lambda_{\mathrm{lc}} \int_{\lambda_{\mathrm{m}}}^{\lambda_{\mathrm{M}}} \mathrm{d} \lambda\left(I(\lambda)-I_{\mathrm{c}}\right)}-1\right),
$$

where $\lambda_{\mathrm{m}}$ and $\lambda_{\mathrm{M}}$ are minimum and maximum wavelengths of the profile, and $I_{\mathrm{c}}$ is the continuum intensity. Here $\lambda_{\mathrm{m}}$ and $\lambda_{\mathrm{M}}$ are $-0.75 \AA$ and $+0.75 \AA$ from the line center, respectively.

\subsubsection{Doppler Velocity 6: Line Center (Full)}

The Doppler velocity of the line center of the full line profile is determined by the location of the minimum of a fourth-order polynomial fit over the range $\pm 32 \mathrm{~m} \AA$ around the minimum-intensity wavelength.

\subsubsection{Doppler Velocity Maps}

Samples of these velocity maps are shown in Figure 5 (raw STAGGER and MURaM) and Figure 6 (STAGGER with the resolution reduced by the HMI PSF and HMI observation). For the HMI observation datasets, we lack the center-ofgravity and the line-center Doppler velocities from the full spectra because we only have filtergrams. Instead, we show the standard HMI-algorithm Dopplergram provided by the pipeline as well. The contrasts in the different Dopplergram observables are different. This suggests that we might have information from 
various heights. It is clear from the comparison between Figures 5 and 6 that the contrast of the Doppler velocity is significantly reduced after smoothing with PSF, although the differences in contrast between the different Dopplergram observables are still present. In Figure 6 the gray-scale contrast, or the dynamic range, of the observation Dopplergrams is less than the synthesized one. This may also indicate the difference between the theoretical PSF that we used and the real PSF for HMI instruments.

\section{Comparison of the Synthetic Doppler Velocities and the Original Velocity Field in the Simulation Box}

Following Fleck, Couvidat, and Straus (2011), we estimate the contribution layer height of synthetic "multi-height" Dopplergrams, by calculating the correlation coefficients of the line-of-sight velocity derived from the synthetic Dopplergrams and the original vertical velocities $\left[v_{z}\right]$ in the simulation box. These correlations are shown in Figure 7. The heights where the correlation coefficients of the Doppler velocities and the vertical velocity in the atmosphere attain their maxima are summarized in Table 2. For this calculation, we use one snapshot of each simulation dataset. To estimate the errors in the maximum-correlation heights, we subdivide the field of view into nine areas and calculate the standard deviations of the heights of maximum correlation coefficients. Note that the FOV of MURaM data is $9 \mathrm{Mm}$ square while that of STAGGER is $48 \mathrm{Mm}$ square, and this causes larger standard deviations of the heights in MURaM datasets. In this case the subdivided area in MURaM FOV is about $1 \mathrm{Mm}$, namely, about the size of the granular cells. The large standard deviation of the height, therefore, indicates the spatial variation due to the granular cells. In Table 2 we also show the results obtained from $C^{5}$ BOLD for reference; these results are from further analysis of the datasets used in Fleck, Couvidat, and Straus (2011). According to this comparison, if the resolutions are similar, the contribution-layer heights in different atmospheric models are similar.

Figure 8 shows that the auto-correlation coefficient of $v_{z}$ has a broad peak and its FWHM is about $500 \mathrm{~km}$, i.e., several scale heights. Therefore, this is consistent with what is shown in Figure 7; the Dopplergrams of this wavefield should have a broad range of contribution layers.

Fleck, Couvidat, and Straus (2011) show (in their Figure 5) the correlation coefficient between the velocity derived from the HMI filtergrams and $v_{z}$ in their simulation box. They found that the normal HMI-algorithm Dopplergram and the center of gravity of the line have a peak correlation with the true $v_{z}$ around $100 \mathrm{~km}$ above the surface, while the Doppler shift of the line center has a peak correlation around $230 \mathrm{~km}$. These results are consistent with our findings. Also, Fleck, Couvidat, and Straus (2011) discuss the effects of spatial resolution. Their original horizontal grid size is $14 \mathrm{~km}$, and if they apply the estimated HMI PSF to their data, the contribution height increases by about $50 \mathrm{~km}$ for their synthetic HMI-algorithm Dopplergram. On the other hand, our results show that the contribution layer heights are $70-80 \mathrm{~km}$ higher if we apply the HMI PSF. Since the PSF in Fleck, Couvidat, and Straus (2011) is Gaussian with FWHM 

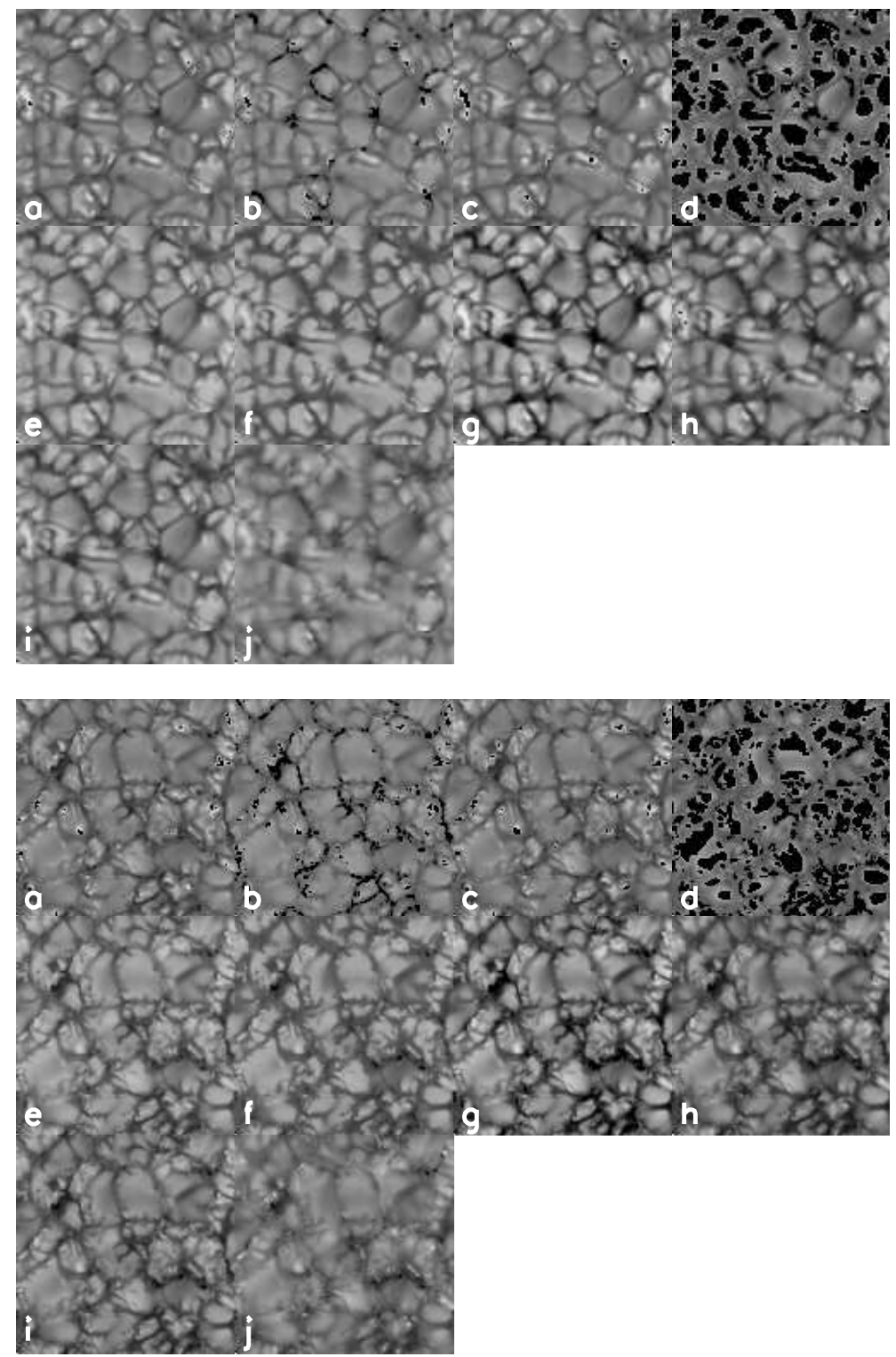

Figure 5. Sample synthetic Dopplergrams obtained from the STAGGER simulations (top) and from the MURaM simulations (bottom): average-wing (a), far-wing (b), wing (c), core (d), center of gravity from filtergrams (e), line center from filtergrams (f), first simplified HMI-algorithm Dopplergram (g), second simplified HMI-algorithm Dopplergram (h), center of gravity from full spectra (i), and line center from full spectra (j). The areas and the field of view are identical to those of Figure 3 (top: $10 \mathrm{Mm}$ square, bottom: $9 \mathrm{Mm}$ square). The gray scale range is from -5 (black, downflow) to $+5 \mathrm{~km} \mathrm{~s}^{-1}$ (white, upflow). Note that in the panels (b) - (d), especially in (d), there are many unusable points (in black) because of the limited applicable range (see Appendix $\mathrm{A}$ for details). 

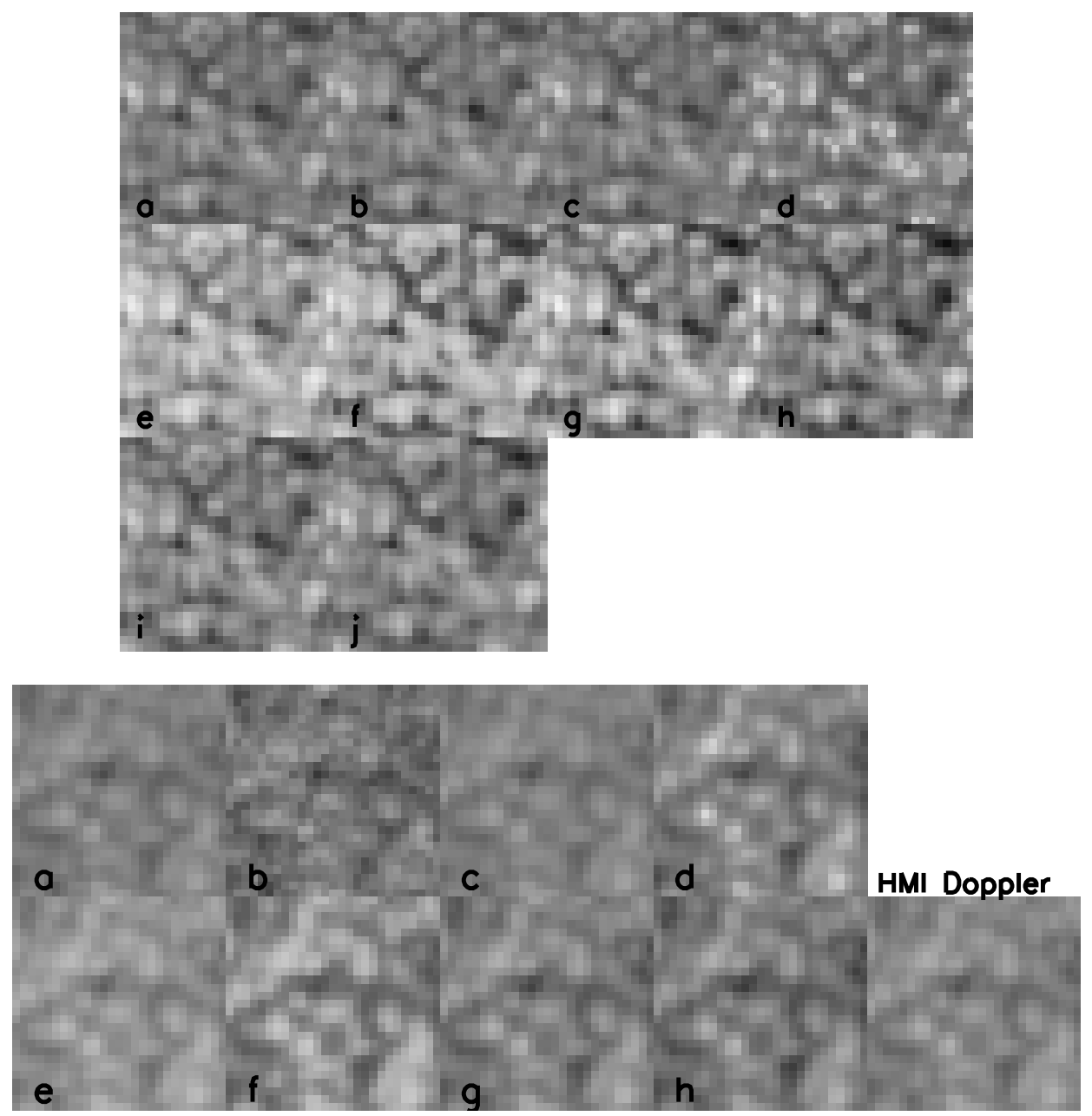

Figure 6. Sample synthetic Dopplergrams obtained from STAGGER datasets with the resolution reduced by the approximate HMI PSF (top) and obtained from HMI observations (bottom): average-wing (a), far-wing (b), wing (c), core (d), center of gravity from filtergrams (e), line center from filtergrams (f), first simplified HMI-algorithm Dopplergram (g), second simplified HMI-algorithm Dopplergram (h), center of gravity from full spectra (i), and line center from full spectra (j). The area of each map is $10 \mathrm{Mm}$ squre and the fields of view are identical to Figure 4 The gray-scale range in this figure is from -2.5 (black, downflow) to +2.5 $\mathrm{km} \mathrm{s}^{-1}$ (white, upflow), which is smaller than that in Figure 5 The contrast of the Doppler velocity is significantly reduced by the PSF smoothing. The HMI-algorithm Dopplergram is also shown in the bottom right panel.

of $1.5 \operatorname{arcsec}(\approx 3$ HMI pixels), the smearing is more significant than our PSF (Equation (1)). Why more smearing causes a smaller shift of contribution layer heights is still unclear. This difference might be caused by other properties, such as differences in atmospheric profiles or convection models.

As shown in Table 2 the contribution layer of the line-center Dopplergrams derived from the full spectra is higher than those derived from the filtergrams. 


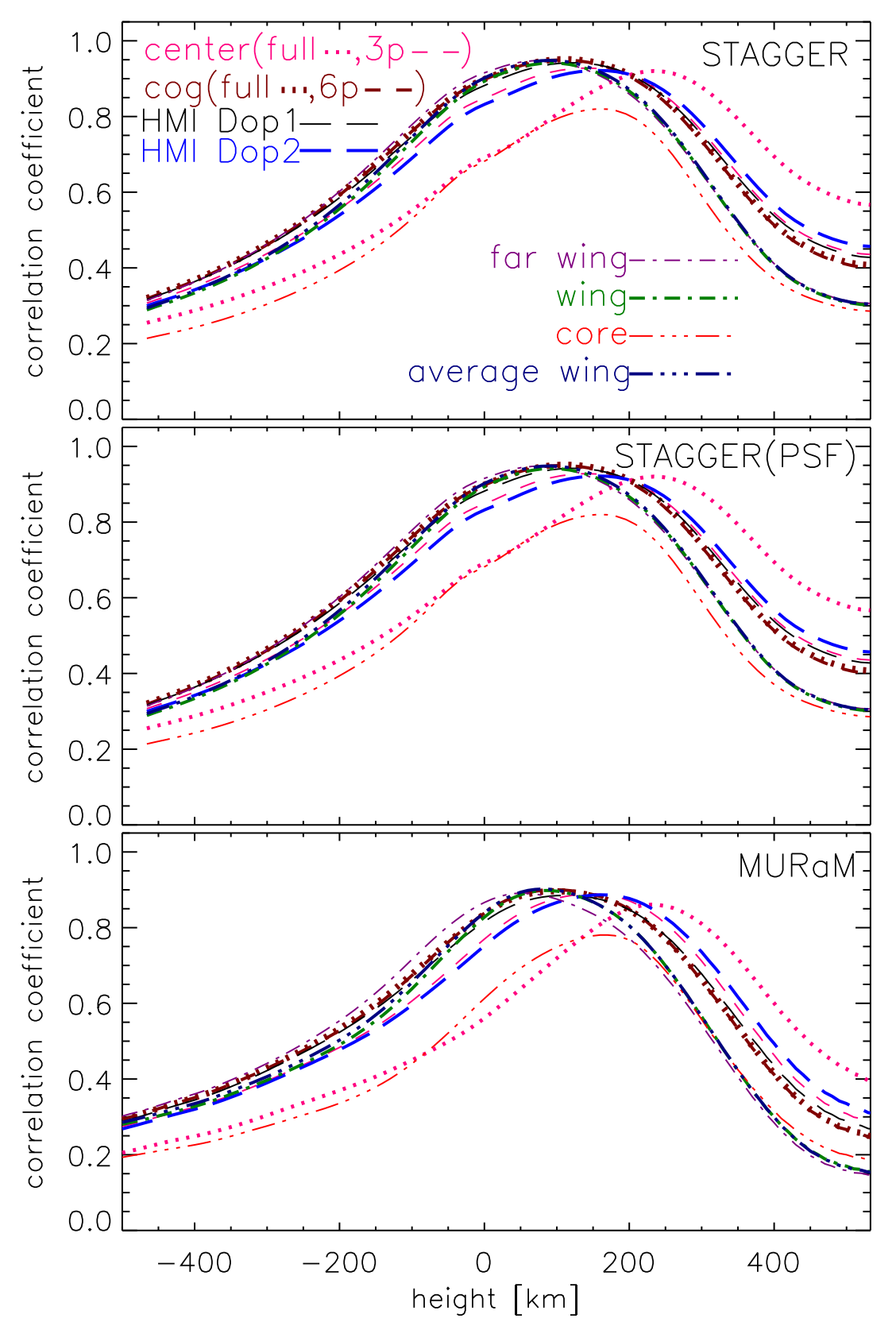

Figure 7. Correlation coefficients of the synthetic Doppler velocities and the original $v_{z}$. The top, middle, and bottom panels are from STAGGER data with the original resolution, STAGGER data with reduced resolution (HMI PSF), and MURaM data, respectively. The dash-dotted curves indicate the simple Dopplergrams derived from the Doppler signals made of pairs of filtergrams, namely, the core, wing, far-wing, and average-wing Doppler velocities. The dashed curves indicate the velocities derived from the filtergrams (center-of-gravity, or cog, line-center, and the first and second simplified HMI-algorithm Dopplergrams), while the dotted curves indicate the velocities derived from the full spectra (center of gravity and line center). 
Table 2. Heights where the correlation coefficient between the Doppler velocity and $v_{z}$ in the atmosphere are maximum. The Doppler velocities listed in the titles of the rows are defined in Section 2.3 The numbers in the parentheses are the standard deviations of the heights of maximum correlation coefficients of nine non-overlapping subareas. Note that the FOV of MURaM data is $9 \mathrm{Mm}$ square while that of STAGGER is $48 \mathrm{Mm}$ square.

\begin{tabular}{lllll}
\hline & & \multicolumn{2}{c}{ Simulated data } \\
Type of Doppler velocity & $\begin{array}{l}\text { STAGGER } \\
{[\mathrm{km}]}\end{array}$ & $\begin{array}{l}\text { STAGGER w/ } \\
\text { PSF }[\mathrm{km}]\end{array}$ & $\begin{array}{l}\text { MURaM } \\
{[\mathrm{km}]}\end{array}$ & $\begin{array}{l}\text { CO }^{5} \text { BOLD } \\
{[\mathrm{km}]}\end{array}$ \\
\hline Line center (full) & $234(6.5)$ & $260(7.8)$ & $230(34)$ & 230 \\
Core & $157(4.3)$ & $208(4.3)$ & $170(68)$ & \\
2nd HMI-algorithm & $157(4.3)$ & $221(6.5)$ & $160(64)$ & 140 \\
Line Center (3pts) & $144(0.0)$ & $221(8.6)$ & $150(68)$ & 125 \\
1st HMI-algorithm & $118(5.7)$ & $195(6.8)$ & $110(56)$ & 100 \\
Center of gravity (full) & $118(6.8)$ & $182(6.8)$ & $100(58)$ & 90 \\
Center of gravity (6pts) & $105(0.0)$ & $195(9.2)$ & $100(43)$ & 90 \\
Wing & $92(0.0)$ & $170(6.5)$ & $90(31)$ & 80 \\
Average-wing & $92(0.0)$ & $170(6.5)$ & $80(31)$ & 70 \\
Far-wing & $79(4.3)$ & $157(9.4)$ & $50(47)$ & 55 \\
\hline
\end{tabular}

The contribution-layer height is sensitive to the wavelength range used for the fitting. As we describe in Section 2.3 , we choose $\pm 32 \mathrm{~m} \AA$ around the minimumintensity wavelength, but if we choose a broader range, the contribution-layer height is reduced.

\subsection{Rather Robust Multi-Height Velocity Datasets}

The results of this section tell us that we have several ways to derive multiheight velocity information in the solar atmosphere from the HMI filtergrams. We need to consider, however, not only the formation height of each velocity but also the availability or noise level of the observables. As was discussed by Nagashima et al. (2013), velocities derived from simple Doppler signals $\left[D_{b r}\right]$ (Doppler velocities 1 in Section 2.3) have many unusable points due to the limited wavelength separation of the blue and red pair. For example, for the core pair the blue and red wings are only $34 \mathrm{~m} \AA$ away from the line center. Therefore, if the velocity exceeds $1.7 \mathrm{~km} \mathrm{~s}^{-1}$, the line center is outside of this pair, and the Doppler signal made from the pair is no longer useful to measure the Doppler velocity. Based on the results of this section and the availability of observables, for rather robust multi-height velocity datasets we propose choosing the average-wing Dopplergrams, the HMI-algorithm Dopplergrams, and the linecenter Dopplergrams defined in Section 2.3 .

\section{Phase Difference}

Using these multi-height Dopplergrams, we calculate the phase differences among them. 

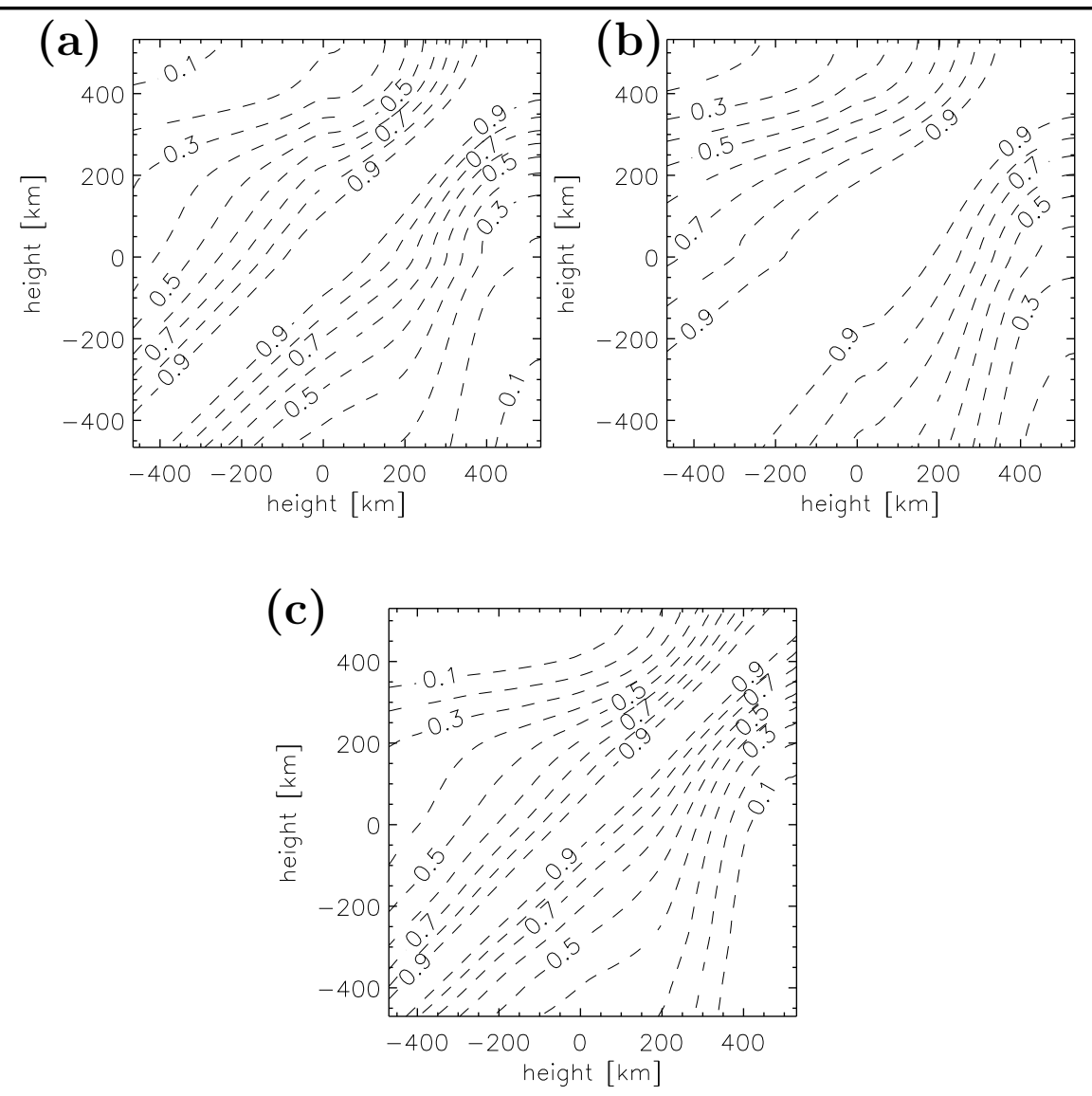

Figure 8. Autocorrelation coefficients of $v_{z}$ in the atmosphere in the simulation boxes: STAGGER datacubes with original resolution (a), STAGGER datacubes with resolution reduced by the HMI PSF (b), and MURaM datacube with original resolution (c).

\subsection{Observed Phase Difference}

For the phase analyses of the HMI observation here, the average-wing and the line-center Doppler velocities are calculated from the six HMI observation filtergrams in the same manner as described in Section 2.3. while as for the HMI-algorithm Dopplergrams, we use the standard product of the HMI pipeline (Couvidat et al. 2012).

Given Fourier transform $\tilde{f}(\boldsymbol{k}, \omega) \equiv|\tilde{f}| e^{\mathrm{i} \phi_{f}}$ and $\tilde{g}(\boldsymbol{k}, \omega) \equiv|\tilde{g}| e^{\mathrm{i} \phi_{g}}$ of two Doppler velocities, $f(\boldsymbol{x}, t)$ and $g(\boldsymbol{x}, t)$, where $\boldsymbol{k}=\left(k_{x}, k_{y}\right)$ is the spatial wavenumber, $\omega$ is the temporal wavenumber, and $\boldsymbol{x}=(x, y)$ and $t$ are the location and the time, respectively, and $\phi_{f}, \phi_{g}$ are real, we define the cross spectrum as $\tilde{f}(\boldsymbol{k}, \omega) \tilde{g}^{*}(\boldsymbol{k}, \omega)=|\tilde{f}||\tilde{g}| e^{\mathrm{i}\left(\phi_{f}-\phi_{g}\right)}$. For Figure 9 we azimuthally average $\tilde{f} \tilde{g}^{*}$ in the $k_{x}-k_{y}$ plane, and calculate the phase of the average as a function of horizontal wavenumber $[k \equiv\|\boldsymbol{k}\|]$ and frequency $[\nu=\omega /(2 \pi)]$. In what follows the phase of 
the azimuthally averaged $\tilde{f} \tilde{g}^{*}$ is denoted by simply $\phi_{f}-\phi_{g}$. This $\phi_{f}-\phi_{g}$ ranges from $-\pi$ to $+\pi$. We also calculate the coherence to show how reliable the phase has been determined. Here we define the coherence of the two velocity fields $[f$ and $g]$ as $\left|\left\langle\tilde{f} \tilde{g}^{*}\right\rangle\right| /\left(\left\langle|\tilde{f}|^{2}\right\rangle\left\langle|\tilde{g}|^{2}\right\rangle\right)^{1 / 2}$, where \langle\rangle means azimuthal average at each $k$.

Figure 9 shows the phase differences between the average-wing, the HMIalgorithm, and the line-center Dopplergrams, as well as their coherences. For this calculation, we use 512 frames with 45 -second cadence (384 minutes) starting from 4:17:15 UT on 23 January 2011. The region is a 30-square-degree quiet region near the disk center. This region is tracked at the Carrington rate by using mtrack (Bogart et al. 2011), and the central point of the area passes the heliographic disk center at the mid-point of the time period. Mtrack is a module used in the HMI pipeline to make tracked and mapped datacubes at selected heliographic coordinates.

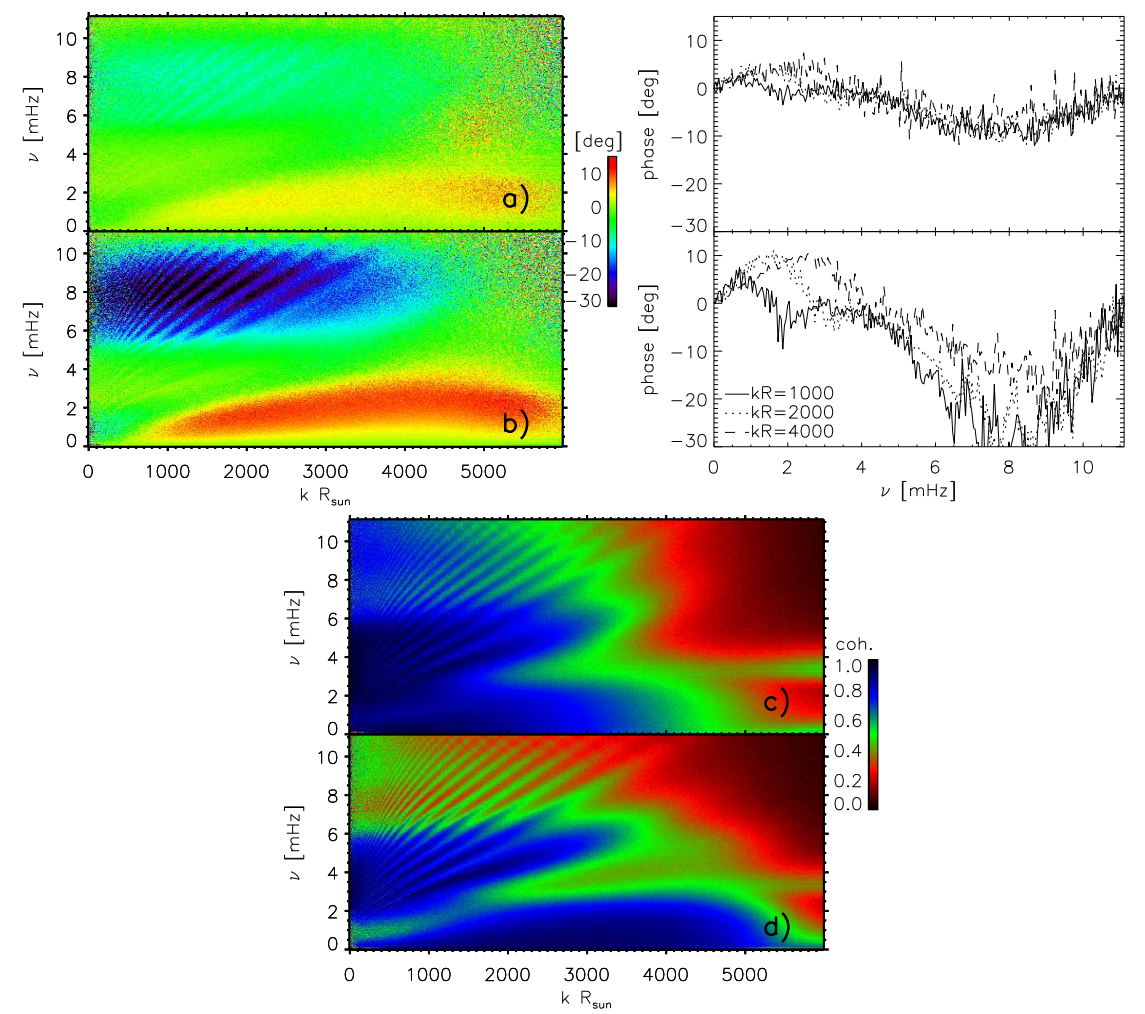

Figure 9. Top: Observed phase difference between the line-center and HMI-algorithm Dopplergrams $\left[\phi_{\text {center }}-\phi_{\mathrm{HMI}}\right]$ (a) and between the line-center and average-wing Dopplergrams $\left[\phi_{\text {center }}-\phi_{\text {average-wing }}\right](\mathrm{b})$. The horizontal wavenumber $[k]$ in the horizontal axis in the left panel is normalized by the solar radius $\left[\mathrm{R}_{\odot}\right]$. Slices at $k \mathrm{R}_{\odot}=1000,2000,4000$ are shown in the top-right panel. Bottom: coherence spectra of the line-center and HMI-algorithm Dopplergrams (c), and the line-center and average-wing Dopplergrams (d).

The phase difference shows three characteristic features in $k-\omega$ space as we expect. First, in the $p$-mode regime (lower frequency, lower wavenumber), the phase difference is nearly zero because they are eigenmodes of the Sun. Sec- 
ond, above the acoustic-cutoff frequency in the photosphere $(\approx 5.4 \mathrm{mHz})$ the phase difference is negative and proportional to the frequency which indicates upward-propagating waves. Third, in the convective regime, namely, in the lower frequency and the larger wavenumber ranges, the phase difference is positive. This is a signature of atmospheric gravity waves, which was reported, e.g., by Straus et al. (2008) for CO ${ }^{5}$ BOLD datasets and by Straus et al. (2009) in observations. Note that in the highest frequency range $(\nu>8 \mathrm{mHz})$ the phase difference cannot be trusted because of their proximity to the Nyquist frequency and possible aliasing effects. The coherence spectra show that these characteristic phase-difference features are reliable over most of $k-\omega$ space, in particular in the $p$-mode and internal-gravity wave regimes. At high wave numbers $\left(k R_{\odot} \gtrsim 4000\right)$ and high frequencies (red areas in the coherence diagrams), the measured phase signal becomes unreliable (also note the scatter of the phase signal in this area in the $k-\omega$ phase diagrams). It is beyond the scope of this article to discuss the differences between the pseudo $p$-mode ridges and the surrounding inter-ridges, which remain a puzzle, in both the phase-difference and coherence spectra.

The phase differences above the acoustic-cutoff frequency in the photosphere $(\approx 5.4 \mathrm{mHz})$ increase linearly as the frequency increases. Around $8 \mathrm{mHz}$, the phase differences $[\Delta \phi]$ are

$$
\begin{aligned}
\Delta \phi_{\text {center-HMI }} & =\phi_{\text {center }}-\phi_{\mathrm{HMI}} \approx-10^{\circ} \\
\Delta \phi_{\text {center-average-wing }} & =\phi_{\text {center }}-\phi_{\text {average-wing }} \approx-30^{\circ} .
\end{aligned}
$$

This minus sign of the phase means upward propagation. The phase of the line-center Dopplergram is smaller, and, therefore, the layer indicated by the linecenter Dopplergram is higher than the layers indicated by the HMI-algorithm Dopplergram and average-wing Dopplergram. If we simply estimate the height difference between the two formation layers $[\Delta z]$ from $\Delta \phi$ by $\Delta z / c_{\mathrm{s}}=-\Delta \phi /(2 \pi \nu)$ where $c_{\mathrm{s}} \approx 7 \mathrm{~km} \mathrm{~s}^{-1}$ is the photospheric sound speed, we obtain

$$
\begin{aligned}
\Delta z_{\text {center-HMI }} & =-c_{\mathrm{s}} \Delta \phi_{\text {center-HMI }} /(2 \pi \nu) \approx 24 \mathrm{~km} \\
\Delta z_{\text {center-average-wing }} & =-c_{\mathrm{S}} \Delta \phi_{\text {center-average-wing }} /(2 \pi \nu) \approx 73 \mathrm{~km} .
\end{aligned}
$$

The height difference $\Delta z_{\text {center-HMI }} \approx 24 \mathrm{~km}$ is similar to the height difference between the estimated layers where the velocity signal based on the line-center (three points) and the first HMI-algorithm Dopplergrams form (26 km, see Table 2 , while $\Delta z_{\text {center-average-wing }} \approx 73 \mathrm{~km}$ corresponds to the estimated height difference between the layers where the velocity signal based on the line-center (three points) and average-wing Dopplergrams form (52 km, see Table 2). However, they are not necessarily identical to each other, because the phase difference is measured at a particular wave mode $(k, \omega)$, while the height measured by taking the correlation coefficients in Section 3 shown in Table 2 is for the total velocity field. This is further discussed in the next subsection.

\subsection{Velocity Response Function}

The vertical velocity discussed in Section 3 is a superposition of turbulentconvective and wave motions, and the height at which the correlation between the 
velocity and Doppler signal is highest is based on both the wave and convective motions. In this section we discuss the velocity response functions. If the velocities due to the waves were small compared to the convective motions, then the height at which the Doppler signal is affected by the wave motions is determined by the response function (and the vertical component of the wave velocities). If the velocities due to the waves turn out to be substantial, the response functions therefore do not allow the height range to be cleanly determined where the Doppler signal is influenced by the waves. None the less, the response functions give some insight into the problem.

We computed the velocity response function by a program to compute full STOkes PROfiles of Zeeman split atomic and molecular absorption lines in LTE (STOPRO) included in the SPINOR code (Frutiger et al., 2000). The response function $\left[R\left(\lambda, \tau, v_{z}\right)\right]$ is the function indicating how the vertical-velocity field perturbation in each layer (in terms of optical depth $[\tau]$ ) affects the intensity, and is defined by

$$
I\left(\lambda, v_{z}^{\prime}\right)-I\left(\lambda, v_{z}\right)=I_{\text {cont }} \int \mathrm{d} \tau R\left(\tau, \lambda, v_{z}\right)\left[v_{z}^{\prime}(\tau)-v_{z}(\tau)\right]
$$

where $I\left(\lambda, v_{z}\right)$ is the intensity at the wavelength $\lambda$ if the vertical velocity field is $v_{z}=v_{z}(\tau), v_{z}^{\prime}(\tau)$ is the perturbed vertical velocity, and $I_{\text {cont }}$ is the continuum intensity. The response function thus gives the linear sensitivity of the intensity to a small change in the velocity field.

Figure 10 shows the response functions. The function is calculated at each pixel in a snapshot and averaged over a 10-Mm-square area of STAGGER datacubes convolved with the HMI filter profiles. To make this figure, using the conversion of the optical depth $[\tau]$ into the geometrical height $[z]$, we plot $R\left(\tau, \lambda, v_{z}\right) \mathrm{d} \tau / \mathrm{d} z$ as a function of $z$. From Figure 10 it is clear that the sensitivity of the far-wing pair $\left[I_{0}\right.$ and $\left.I_{5}\right]$ to the velocity is low, and the far-wing Dopplergrams might not be reliable.

For simplicity, here we consider the simple Dopplergram cases only (Doppler velocities 1 defined in Section 2.3) using these response functions. If we assume the denominator of $D_{\mathrm{br}}=\left(I_{\mathrm{b}}-I_{\mathrm{r}}\right) /\left(I_{\mathrm{b}}+I_{\mathrm{r}}\right)$ does not have much dependence on the velocity, the response function for $D_{\mathrm{br}}$ is $R_{\mathrm{br}} \approx R\left(\tau, \lambda_{\mathrm{b}}, v_{z}\right)-R\left(\tau, \lambda_{\mathrm{r}}, v_{z}\right)$. These $R_{\mathrm{br}}$ are shown in Figure 10 as well. The heights of the center of gravity of $R_{\mathrm{br}}$ are $140 \mathrm{~km}$ for average-wing and $210 \mathrm{~km}$ for core, and the formation height difference between average-wing and the core is $70 \mathrm{~km}$. Although this simple core Doppler velocity is not identical to the line-center Doppler velocity, here we may use the core Doppler velocity as a proxy for the line center. Then, this is consistent with the rough estimate of the height difference derived from the earlier phase-difference measurement, $\Delta z_{\text {center-average-wing }} \approx 73 \mathrm{~km}$.

\subsection{Simulated Phase Difference}

To compare with the observed phase differences, we calculate the phase differences in several ways using the STAGGER simulation. The oscillation power in the HMI observations and the STAGGER simulation are shown in Figure 11. Although the power contrast of the $p$-mode peaks is weaker in the STAGGER 


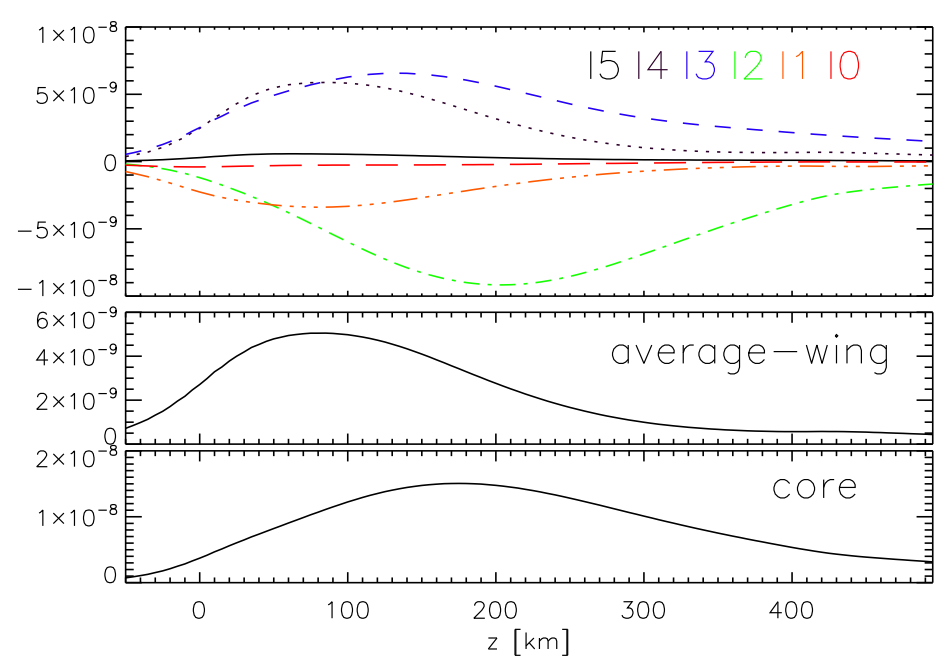

Figure 10. Velocity response functions convolved with the HMI filter profiles (shown in Figure 1. made from STAGGER simulation dataset. The functions are calculated by the SPINOR code for each pixel and then averaged over an area with $10 \mathrm{Mm}$ square. The units of the response functions are $\left(\mathrm{cm} \mathrm{s}^{-1} \mathrm{~km}\right)^{-1}$. The top panel shows the response functions for the filtergrams (I0: red long-dashed, I1: orange dash-triple-dotted, I2: green dash-dotted, I3: blue short-dashed, I4: dark-purple dotted, I5: black solid), while the bottom two panels show the response functions for the average-wing and core Doppler velocities.

simulation, the general trends agree with each other. In particular, both spectra have power humps in the convection/internal-gravity wave regime, namely, in the lower-frequency range.

We calculate the phase difference of the 221-minute-long STAGGER simulation data time series with one-minute cadence in the following ways.

\subsubsection{Phase Difference 1: Dopplergrams from Synthesized Filtergrams}

We use the same line-center, HMI-algorithm, and the average-wing Doppler velocities as those used in the observation data plot (Figure 9). The phase differences between them are shown in Figure 12

\subsubsection{Phase Difference 2: Dopplergrams from Synthesized Spectra}

Although only filtergrams rather than full spectra are obtained from the HMI observations, here for comparison we use the center-of-gravity and line-center Doppler velocities calculated from the full synthetic spectra, which are defined in Section 2.3. The phase difference between them is shown in Figure 13 . According to the results in Section 3 and those of Fleck, Couvidat, and Straus (2011), the contribution height of the center-of-gravity Dopplergram is very similar to the first HMI-algorithm Dopplergram, although, according to Table 2, the contribution heights of the line-center Dopplergram made from the full spectrum is much higher than that made from filtergrams. Therefore, this figure is a counterpart of Figure $12 \mathrm{a}$. It is not a surprise that the absolute values of phase difference 


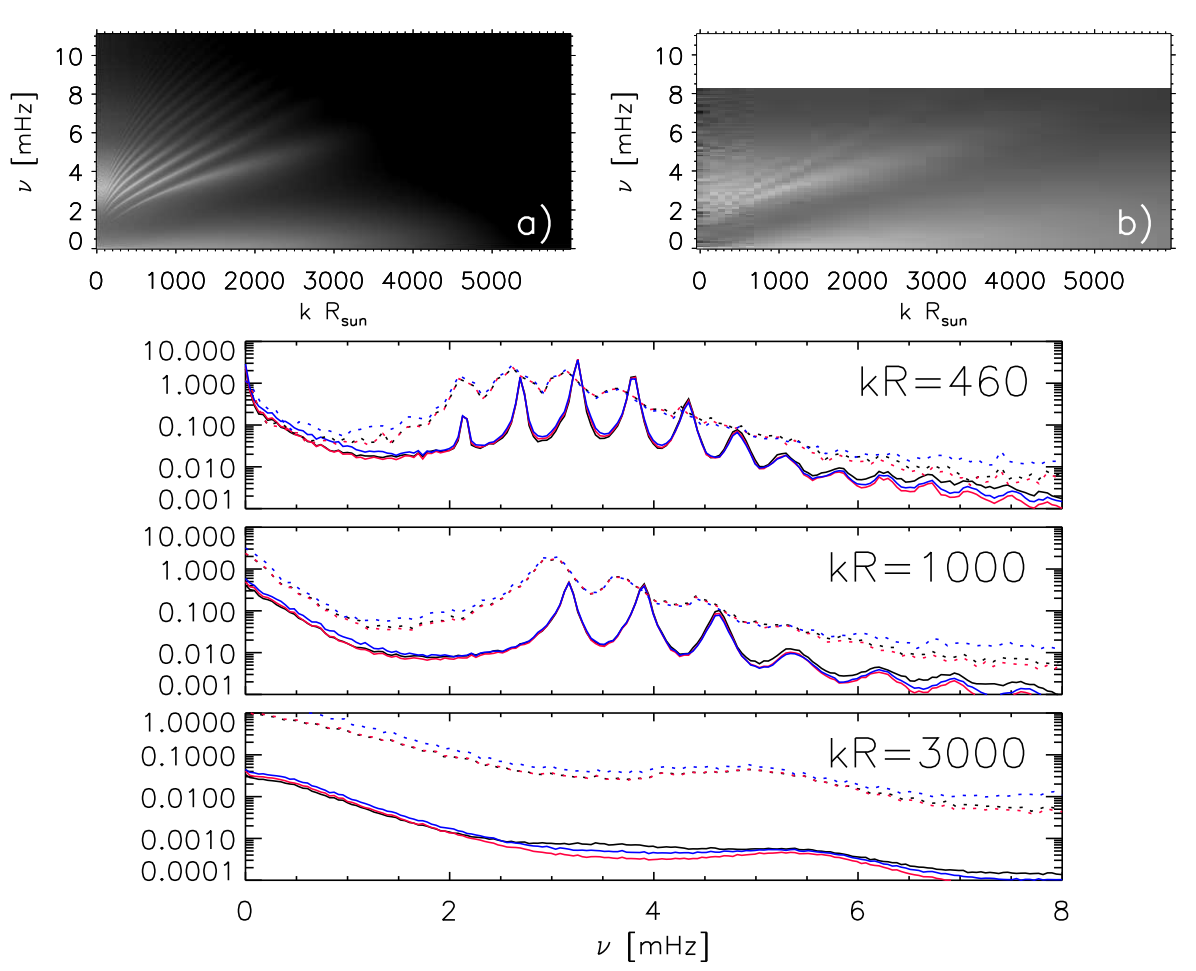

Figure 11. Power spectra of the HMI-algorithm Dopplergrams made from HMI observation data (a) and from STAGGER simulation data (b) in logarithmic gray scaling (white indicates higher power, while black indicates lowest power). The observation and simulated datasets are identical to what we use for Figures 9 and 12 respectively. Note that the Nyquist frequency of the simulation data (b) is lower than that of the observation (a), because of the difference in cadence $(\Delta t=60$ seconds instead of 45 seconds). The bottom panel shows slices at several $k \mathrm{R}_{\odot}$. The solid and dotted curves indicate the observations and STAGGER data. Line center, HMI-algorithm Dopplergram, and average-wing Doppler velocities are in black, red, and blue, respectively. Each power spectrum is normalized by its average power in the region $100 \leq k \mathrm{R}_{\odot} \leq 500$ and $3 \mathrm{mHz} \leq \nu \leq 3.5 \mathrm{mHz}$, and in the power map the grayscale range is from $10^{-4}$ to $10^{2}$

both in the acoustic regime (propagative acoustic waves) and in the convection regime (atmospheric gravity waves) are larger than those in Figure 12p; This is because the height difference between the Dopplergrams made from the full spectrum is larger (see Table 2).

\subsubsection{Phase Difference 3: Vertical Velocities at Iso-Optical-Depth Surfaces}

We choose the layers with the optical depth $\log \tau=(-1.5,-1,-0.75,-0.5)$, and calculate the phase differences of the vertical velocity field $\left[v_{z}\right]$ among these layers. It is not straightforward to estimate the geometrical heights of these layers, because the relationship between the geometrical heights and optical depths significantly varies from point to point. To get a very rough idea, however, we here show the geometrical heights calculated in the average STAGGER atmosphere: $223 \mathrm{~km}(\log \tau=-1.5), 145 \mathrm{~km}(\log \tau=-1), 104 \mathrm{~km}(\log \tau=-0.75)$ 

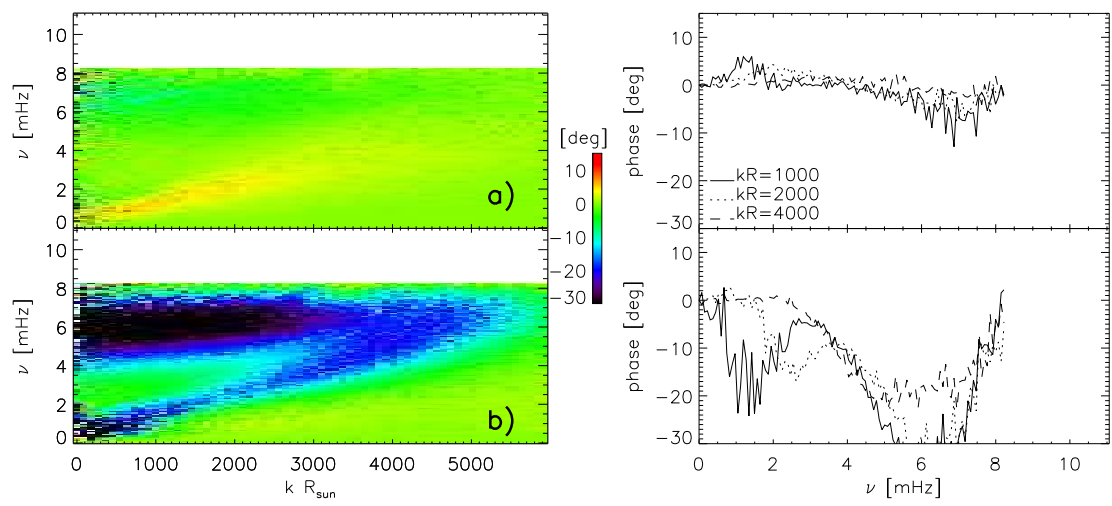

Figure 12. Phase difference between the line-center and first HMI-algorithm Dopplergram, $\phi_{\text {center }}-\phi_{\text {HMI }}(\mathrm{a})$ and between the line-center and average-wing $\phi_{\text {center }}-\phi_{\text {average-wing }}(\mathrm{b})$, from STAGGER simulation data. Slices at $k \mathrm{R}_{\odot}=1000,2000$, and 4000 are shown in the right panel.
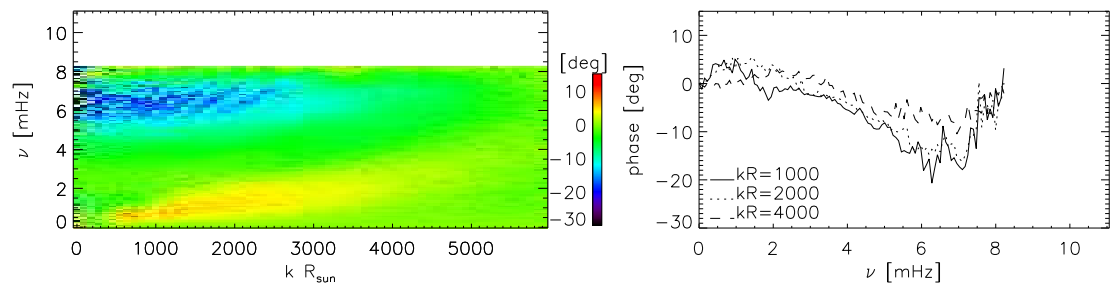

Figure 13. Phase difference between the line-center and center of gravity Dopplergrams $\phi_{\text {center,full }}-\phi_{\mathrm{cog} \text {,full }}$ synthesized from STAGGER simulation data. Note that these are calculated not from the synthesized filtergrams but from the full spectra, namely they are Dopplergrams 5 and 6 defined in Section 2.3 Slices at $k \mathrm{R}_{\odot}=1000,2000$, and 4000 are shown in the right panel. The contribution height of the center-of-gravity Dopplergram is very close to the first HMI-algorithm Dopplergram, and this figure is a counterpart of Figure 12 , although the contribution layer of line-center Dopplergram made from the full spectrum is higher than that made from filtergram, which makes the phase difference larger than Figure 12 .

and $62 \mathrm{~km}(\log \tau=-0.5)$ above the surface $(\log \tau=0)$. Figure 14 shows the phase differences among these layers.

\subsubsection{Phase Difference 4: Vertical Velocities at Iso-Geometrical Height Layers}

We choose four layers [92 km, $118 \mathrm{~km}, 144 \mathrm{~km}$, and $170 \mathrm{~km}$ ] and calculate the phase differences of the vertical-velocity field among these layers. The first three are close to the contribution layers for the bulk velocities of the average-wing, the HMI-algorithm, and the line-center Dopplergrams, which are estimated by the correlation coefficients between the vertical velocity in the atmosphere and the synthetic Doppler velocities (see Table 2). Figure 15 shows the phase differences between these layers. 

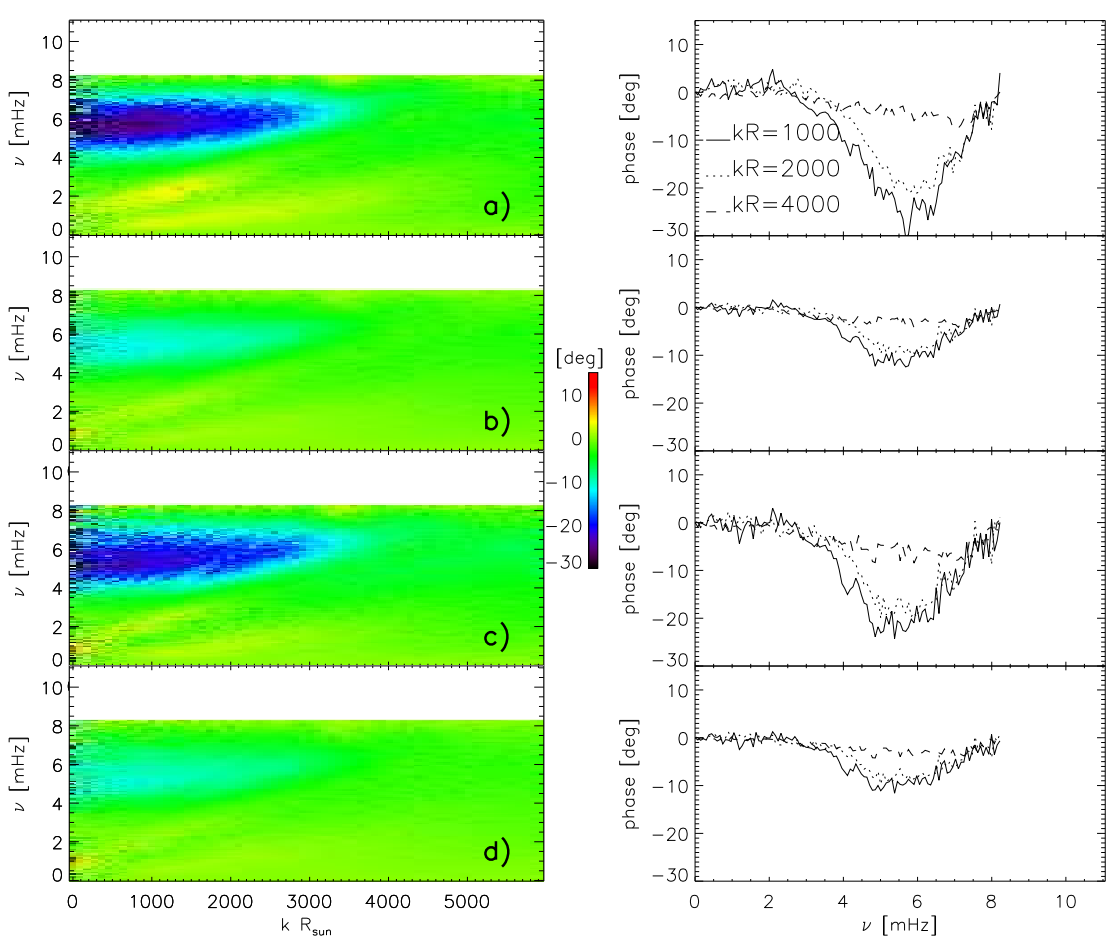

Figure 14. Phase difference of the vertical velocity $\left[v_{z}\right]$ between pairs of iso-optical depth surfaces in STAGGER simulation atmosphere: (a) $\phi(\log \tau=-1.5)-\phi(\log \tau=-1)$, (b) $\phi(\log \tau=-1)-\phi(\log \tau=-0.75)$, (c) $\phi(\log \tau=-1)-\phi(\log \tau=-0.5)$, and (d) $\phi(\log \tau=-0.75)-\phi(\log \tau=-0.5)$. The slices at $k \mathrm{R}_{\odot}=1000,2000$, and 4000 are shown in the right panel. The geometrical heights calculated in the atmosphere averaged over the field of view are $223 \mathrm{~km}(\log \tau=-1.5), 145 \mathrm{~km}(\log \tau=-1), 104 \mathrm{~km}(\log \tau=-0.75)$, and $62 \mathrm{~km}$ $(\log \tau=-0.5)$, although the relationship between the geometrical height and optical depth significantly varies from point to point.

\subsubsection{Summary of Phase Difference}

Generally, above the photospheric acoustic-cutoff frequency, the phase differences found in the HMI observational data and the STAGGER data have similar trends, although the acoustic-cutoff frequency of STAGGER seems lower than that of the Sun. Figure 16 shows the acoustic-cutoff frequency profiles near the surface calculated using the mean STAGGER atmosphere and the Model $\mathrm{S}$ atmosphere. Here the acoustic-cutoff frequency is defined as $\omega_{\mathrm{ac}}=c_{\mathrm{s}} / 2 H_{\rho}$, where $H_{\rho}$ is the density scale height. The profiles of $c_{\mathrm{s}}$ and $H_{\rho}$ are also plotted in Figure 16. The height $z=0$ is defined as the layer where $\tau_{5000} \AA=1$ for each profile see Figure 2). The acoustic-cutoff frequency is slightly lower in the STAGGER atmosphere, which is consistent with what was indicated by the phase difference distributions (Figures 9 and 12 ). However, there is a dip of the acoustic cutoff in the top layers in STAGGER atmosphere. This is due to adding some Newtonian cooling to the energy equation near the top boundary to improve computational stability. 

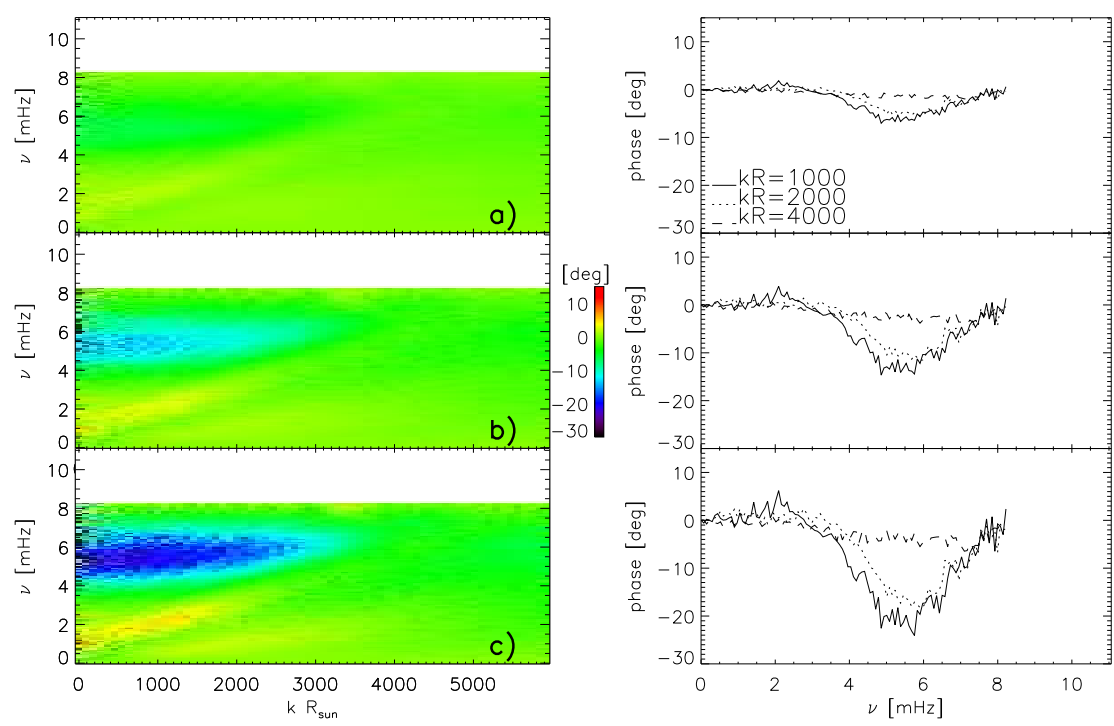

Figure 15. Phase difference of the vertical velocity $\left[v_{z}\right]$ between the layer at $92 \mathrm{~km}$ above the surface and several other layers in STAGGER simulation atmosphere: (a) $\phi(118 \mathrm{~km})-\phi(92 \mathrm{~km})$, (b) $\phi(144 \mathrm{~km})-\phi(92 \mathrm{~km})$, and $(\mathrm{c}) \phi(170 \mathrm{~km})-\phi(92 \mathrm{~km})$. The slices at $k \mathrm{R}_{\odot}=1000,2000$, and 4000 are shown in the right panel.

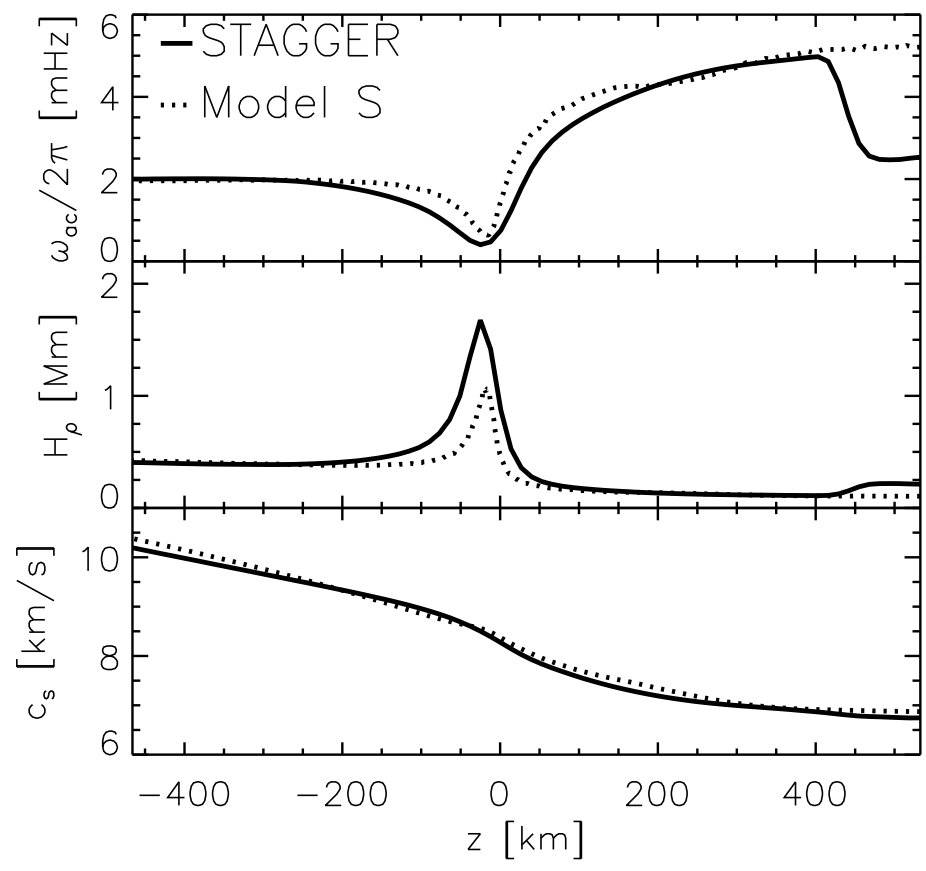

Figure 16. Acoustic cutoff frequency $\left[\omega_{\mathrm{ac}} \equiv c_{\mathrm{S}} /\left(2 H_{\rho}\right)\right]$ (top), density scale height $\left[H_{\rho}\right]$ (middle), and sound speed $\left[c_{\mathrm{S}}\right]$ (bottom) profiles of STAGGER atmosphere (solid) and Model $\mathrm{S}$ (dotted). 
Since from the observations we usually cannot see the layer with a constant geometrical height but the layer with a constant optical depth, we expect that the phase differences of the vertical velocity between the two iso-optical depth surfaces (Figure 14) are more like the observational phase differences (Figure 9) or the phase differences between two synthetic Dopplergrams (Figure 12), rather than the phase differences of the vertical velocity between two iso-geometrical height layers (Figure 15). However, they all look similar to each other, except for Figure 12b. All of the STAGGER phase difference maps have some signatures that are not found in the observation, however. The signatures are located around the boundary of the acoustic- and gravity-wave regimes, and in Figure $12 \mathrm{~b}$ the phase difference is negative and about $-20^{\circ}$, while in other figures the phase difference is positive and less than $5^{\circ}$. What makes these positive- or negative-value ridges there is still unknown.

In the gravity-wave regime, namely in the lower frequency range, all the phase differences of the STAGGER simulation datasets show little atmospheric gravitywave signals, which are seen clearly in the observation data as was mentioned in Section 4.1. In the power maps (Figure 11), some signatures are seen in the gravity-wave regime both in the observational Doppler-velocity datasets and synthetic (STAGGER) Doppler velocity datasets, and this phase-difference signature of atmospheric gravity waves was reported, e.g., by Straus et al. (2008) for $\mathrm{CO}^{5}$ BOLD simulation datasets and by Straus et al. (2009) in observations. One of the differences between STAGGER and $\mathrm{CO}^{5} \mathrm{BOLD}$ simulations is the extent of the atmosphere. The upper boundary lies around $550 \mathrm{~km}$ above the surface in STAGGER atmosphere and around $900 \mathrm{~km}$ in the $\mathrm{CO}^{5}$ BOLDatmosphere. Since there is convection in both simulations, there is power in the lower frequency range, i.e., in the gravity wave regime, but for atmospheric gravity waves the atmospheric extent (about $550 \mathrm{~km}$ ) of STAGGER might not be sufficient. Another possible interpretation is that radiative damping of the short-wavelength waves in the STAGGER simulation is stronger than in the Sun.

Note that the resolution of the STAGGER data used here is the full resolution, but we checked that the PSF does not change the general trend of these phase differences.

\section{Conclusion and Discussion}

We confirm that we can obtain multi-height line-of-sight velocity information in the solar atmosphere from SDO/HMI filtergrams. We suggest average-wing and line-center Dopplergrams as well as the general pipeline product, (first) HMIalgorithm Dopplergrams as rather robust multi-height velocity datasets among the several Doppler velocities defined in Section 2 based on the estimate of the contribution layer heights and the availability of the observables.

In general, multi-height Doppler observations have the potential to help constrain the height variations of the $p$-mode eigenfunctions in the solar atmosphere (see Baldner and Schou (2012), for applications). In addition, multi-height Doppler observations may be helpful for distinguishing convective motions from oscillations, which in turn may be useful to improve the signal-to-noise ratio in helioseismology studies. 
We estimate the contribution layer heights of several synthetic Doppler velocities computed using numerical convection simulation datasets, STAGGER and MURaM. Although the contribution layer is rather broad, the contribution layer heights of the average-wing and the line-center Dopplergrams are $30-40 \mathrm{~km}$ lower and 30-40 km higher compared to the standard HMI-algorithm Dopplergrams, respectively. Note that the height difference between these Dopplergrams is $70 \mathrm{~km}$ at most, which is about half, at best, of the scale height around the surface (see Figure 16). We can obtain multi-height information from these observables, but since we use the filtergrams taken around a single absorption line, the height difference is relatively limited.

HMI observations show clear phase differences between these Dopplergrams at frequencies above the acoustic cutoff frequency. The height difference estimated by the response functions is consistent with the one estimated by the phase differences. HMI observation data also show a clear signature of atmospheric gravity waves in the lower-frequency ranges, while STAGGER simulation data have only a weak signature.

Although in this study we limited ourselves to quiet-Sun data for the sake of simplicity, multi-height velocity information in magnetic regions is also of great interest. Since the spectral-line shape is changed not only by the velocity fields but also by magnetic field, it is not straightforward to analyze such observations and one would need radiative-transfer calculations including the effect of magnetic field.

The formation layer of the SDO/AIA $1600 \AA$ and $1700 \AA$ passbands are estimated in the lower chromosphere around 430 and $360 \mathrm{~km}$ above the surface (see Fossum and Carlsson, 2005). Multi-height Doppler observations from SDO/HMI either alone or together with SDO/AIA or Interface Region Imaging Spectrograph (IRIS) observations will potentially be also useful to understand how much wave energy is transported in the atmosphere and corona (e.g. the review by Reale, 2010 .

Acknowledgments BL acknowledges support from IMPRS Solar System School. BL computed the line profiles from the STAGGER data cubes and the response functions using the SPINOR code. We thank Michiel van Noort, Thomas Straus, and Jesper Schou for helpful discussions and comments. KN and LG acknowledge support from EU FP7 Collaborative Project "Exploitation of Space Data for Innovative Helio-and Asteroseismology" (SPACEINN). LG acknowledges support from DFG SFB 963 "Astrophysical Flow Instabilities and Turbulence" (Project A1). The HMI data used are courtesy of NASA/SDO and the HMI science team. This work was carried out using the data from the SDO HMI/AIA Joint Science Operations Center Data Record Management System and Storage Unit Management System (JSOC DRMS/SUMS). The NSO/Kitt Peak FTS data used here were produced by NSF/NSO. RS acknowledges support by NASA grant NNX12AH49G and NSF grant AGS1141921. The STAGGER calculations were performed on the Pleiades cluster of the NASA Advanced Supercomputing Division at Ames Research Center. The German Data Center for SDO (GDC-SDO), funded by the German Aerospace Center (DLR), provided the IT infrastructure to process the data. 


\section{Appendix}

\section{A. Conversion of the Doppler Signal Made of Pairs of Filtergrams into Doppler Velocities}

For the calculation of the core, wing, far-wing, and average-wing Doppler velocities (Doppler velocity 1 in the list in Section 2.3), we convert the Doppler signal $\left[D_{\mathrm{br}}\right]$ into the line-of-sight velocity, using the parameters we obtain in the following manner: i) calculate the Doppler shift of the line core from $-10 \mathrm{~km}$ $\mathrm{s}^{-1}$ to $+10 \mathrm{~km} \mathrm{~s}^{-1}$, ii) shift the whole average line profile by the amount of the line-core Doppler shift, and calculate the filtergrams by convolving the shifted line profile with the filter profiles, iii) calculate the Doppler signals $\left[D_{\mathrm{br}}\right]$ for each velocity shift, and iv) fit the velocity to a third-order polynomial function of $D_{\mathrm{br}}$ in a certain range of the velocity. The fitting range is chosen so that the third-order polynomial fitting seems reasonably good. Figure 17 shows how the Doppler signals change as the velocity shift changes. The fitting curves are plotted as well. Figure 17 clearly shows the saturation of the Doppler signal with the significantly large Doppler shift. This limits the range useful for the fitting.

The conversion parameters depend on the line profile. The line profiles obtained from STAGGER and MURaM datasets and the HMI reference line profiles are different (see Figure 1), and we calculate the fitting parameters for each line profile. For comparison, the polynomials obtained by the fitting are overplotted in Figure 18. This figure also includes scatter plots of HMI observation Doppler signals averaged over a certain field of view (see below) and the SDO line-of-sight motion towards the Sun. The observation data were obtained on 22-24 January 2011. The 30-degree-square quiet region near the disk center is tracked at the Carrington rate by using mtrack (Bogart et al. 2011 ).

Note that Nagashima et al. (2013) used the spacecraft motion to compute these conversion curves. The spacecraft motion is limited to $\approx 3.5 \mathrm{~km} \mathrm{~s}^{-1}$ (Schou et al. , 2012) and thus the velocity range of the fitting curve available by this method is limited. Therefore, instead, here we use the average line profiles (for STAGGER and MURaM datasets) or HMI reference profiles (for observation datasets) to obtain larger velocity ranges.

\section{References}

Baldner, C.S., Schou, J.: 2012, Effects of Asymmetric Flows in Solar Convection on Oscillation Modes. Astrophys. J. Lett. 760, L1. DOI ADS

Beeck, B., Collet, R., Steffen, M., Asplund, M., Cameron, R.H., Freytag, B., Hayek, W., Ludwig, H.-G., Schüssler, M.: 2012, Simulations of the solar near-surface layers with the CO5BOLD, MURaM, and Stagger codes. Astron. Astrophys. 539, A121. DOI ADS

Bello González, N., Yelles Chaouche, L., Okunev, O., Kneer, F.: 2009, Dynamics of small-scale magnetic fields on the Sun: observations and numerical simulations. Astron. Astrophys. 494, 1091. DOI ADS.

Bello González, N., Franz, M., Martínez Pillet, V., Bonet, J.A., Solanki, S.K., del Toro Iniesta, J.C., Schmidt, W., Gandorfer, A., Domingo, V., Barthol, P., Berkefeld, T., Knölker, M.: 2010, Detection of Large Acoustic Energy Flux in the Solar Atmosphere. Astrophys. J. Lett. 723, L134. DOI ADS

Bellot Rubio, L.R., Borrero, J.M.: 2002, Iron abundance in the solar photosphere. Application of a two-component model atmosphere. Astron. Astrophys. 391, 331. DOI ADS 
(a)
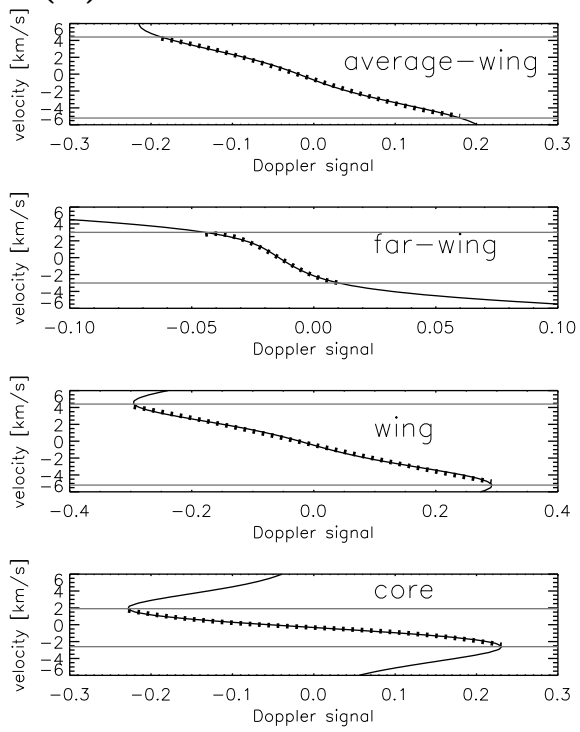

(c)
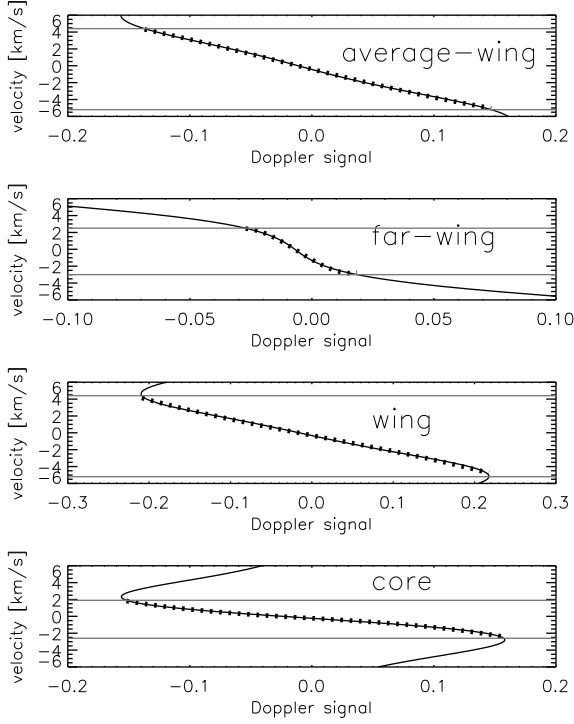

Figure 17. Doppler-signal changes calculated using (a) the STAGGER average line profile, (b) the MURaM average line profile, and (c) the HMI reference line profile. The data points between the solid horizontal lines are used for the fitting, and the fitted third-order polynomial functions are plotted as dots. Positive velocity indicates upward velocity, which causes a blue-shift of the line.

(b)
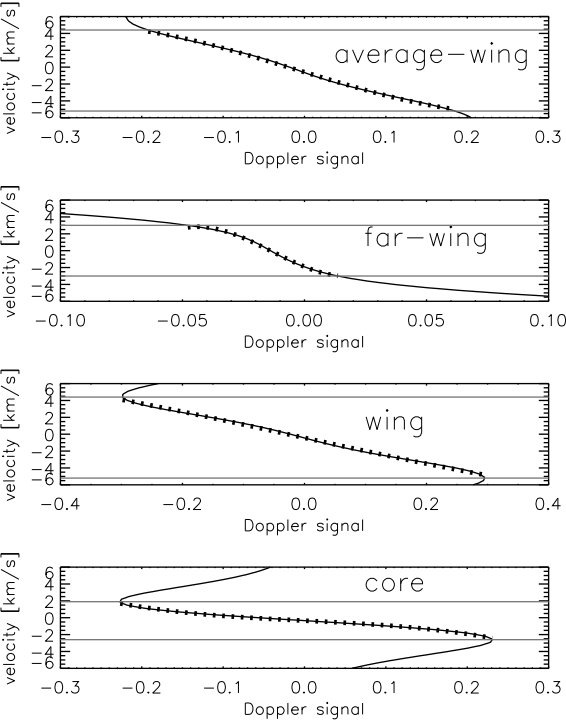

$$
\text { (1) }
$$



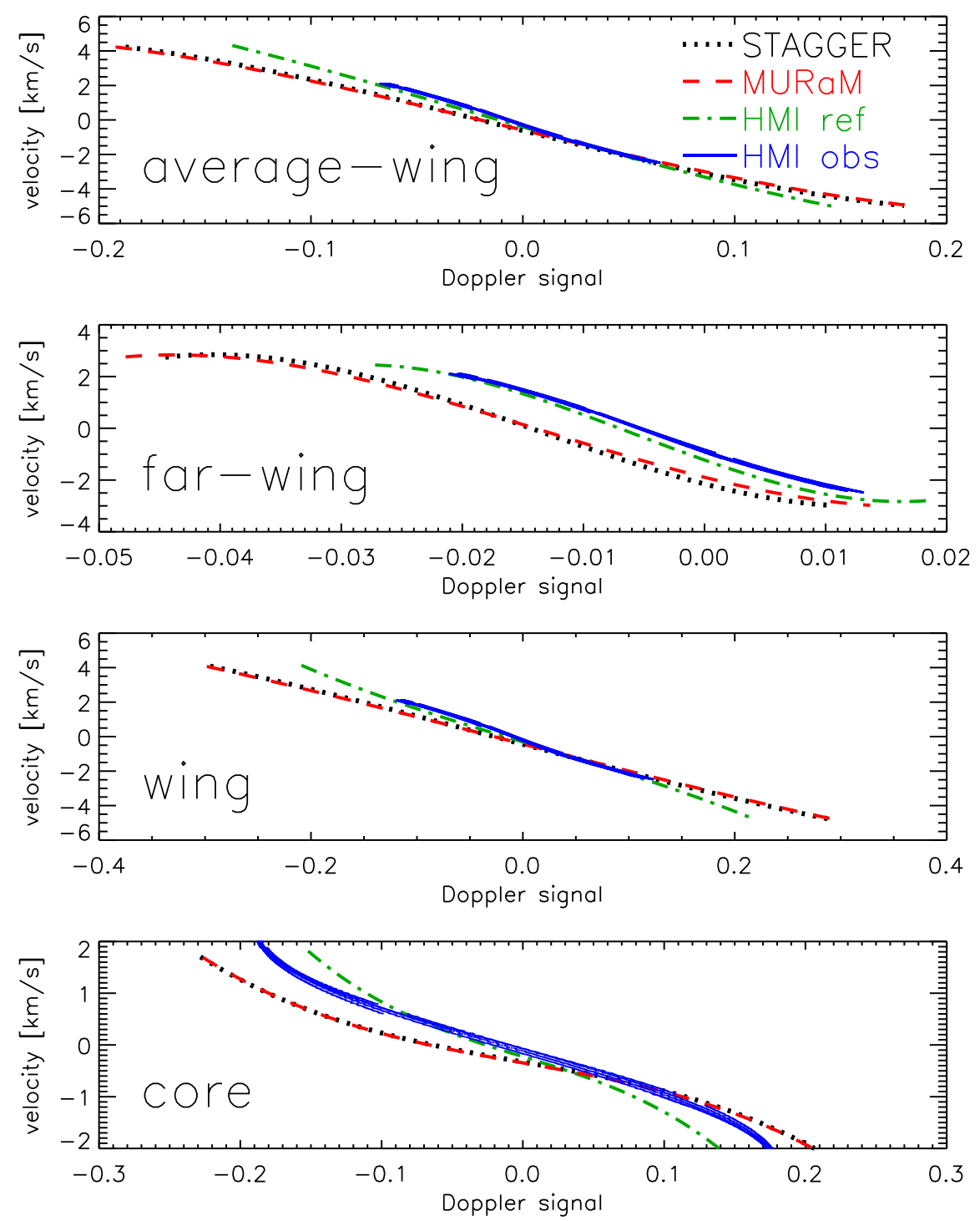

Figure 18. The third-order polynomial fits to the Doppler signals obtained by the fitting to convert the signals to the velocities (black dotted, red dashed, and green dash-dotted curves for each line profile) as shown in Figure 17 Blue dots are the SDO line-of-sight velocity plotted against the mean Doppler signals over the average of the 30-degree-square field of view near the disk center. Positive velocity indicates upward velocity. 
Bogart, R.S., Baldner, C., Basu, S., Haber, D.A., Rabello-Soares, M.C.: 2011, HMI ring diagram analysis I. The processing pipeline. J. Phys. Conf. Ser. 271(1), 012008. DOI ADS

Christensen-Dalsgaard, J., Dappen, W., Ajukov, S.V., Anderson, E.R., Antia, H.M., Basu, S., Baturin, V.A., Berthomieu, G., Chaboyer, B., Chitre, S.M., Cox, A.N., Demarque, P., Donatowicz, J., Dziembowski, W.A., Gabriel, M., Gough, D.O., Guenther, D.B., Guzik, J.A., Harvey, J.W., Hill, F., Houdek, G., Iglesias, C.A., Kosovichev, A.G., Leibacher, J.W., Morel, P., Proffitt, C.R., Provost, J., Reiter, J., Rhodes, E.J. Jr., Rogers, F.J., Roxburgh, I.W., Thompson, M.J., Ulrich, R.K.: 1996, The Current State of Solar Modeling. Science 272, 1286. DOI ADS

Couvidat, S., Rajaguru, S.P., Wachter, R., Sankarasubramanian, K., Schou, J., Scherrer, P.H.: 2012, Line-of-Sight Observables Algorithms for the Helioseismic and Magnetic Imager (HMI) Instrument Tested with Interferometric Bidimensional Spectrometer (IBIS) Observations. Solar Phys. 278, 217. DOI ADS

Fleck, B., Couvidat, S., Straus, T.: 2011, On the Formation Height of the SDO/HMI Fe 6173 A Doppler Signal. Solar Phys. 271, 27. DOI ADS

Fossum, A., Carlsson, M.: 2005, Response Functions of the Ultraviolet Filters of TRACE and the Detectability of High-Frequency Acoustic Waves. Astrophys. J. 625, 556. DOI ADS

Freytag, B., Steffen, M., Dorch, B.: 2002, Spots on the surface of Betelgeuse - Results from new 3D stellar convection models. Astronomische Nachrichten 323, 213. DOI |ADS

Frutiger, C., Solanki, S.K., Fligge, M., Bruls, J.H.M.J.: 2000, Properties of the solar granulation obtained from the inversion of low spatial resolution spectra. Astron. Astrophys. 358, 1109. ADS

Howe, R., Jain, K., Bogart, R.S., Haber, D.A., Baldner, C.S.: 2012, Two-Dimensional Helioseismic Power, Phase, and Coherence Spectra of Solar Dynamics Observatory Photospheric and Chromospheric Observables. Solar Phys. 281, 533. DOI ADS

Jefferies, S.M., McIntosh, S.W., Armstrong, J.D., Bogdan, T.J., Cacciani, A., Fleck, B.: 2006, Magnetoacoustic Portals and the Basal Heating of the Solar Chromosphere. Astrophys. J. Lett. 648, L151. DOI ADS

Kneer, F., Bello González, N.: 2011, On acoustic and gravity waves in the solar photosphere and their energy transport. Astron. Astrophys. 532, A111. DOI. ADS

Lemen, J.R., Title, A.M., Akin, D.J., Boerner, P.F., Chou, C., Drake, J.F., Duncan, D.W., Edwards, C.G., Friedlaender, F.M., Heyman, G.F., Hurlburt, N.E., Katz, N.L., Kushner, G.D., Levay, M., Lindgren, R.W., Mathur, D.P., McFeaters, E.L., Mitchell, S., Rehse, R.A., Schrijver, C.J., Springer, L.A., Stern, R.A., Tarbell, T.D., Wuelser, J.-P., Wolfson, C.J., Yanari, C., Bookbinder, J.A., Cheimets, P.N., Caldwell, D., Deluca, E.E., Gates, R., Golub, L., Park, S., Podgorski, W.A., Bush, R.I., Scherrer, P.H., Gummin, M.A., Smith, P., Auker, G., Jerram, P., Pool, P., Soufli, R., Windt, D.L., Beardsley, S., Clapp, M., Lang, J., Waltham, N.: 2012, The Atmospheric Imaging Assembly (AIA) on the Solar Dynamics Observatory (SDO). Solar Phys. 275, 17. DOI ADS

Mitra-Kraev, U., Kosovichev, A.G., Sekii, T.: 2008, Properties of high-degree oscillation modes of the Sun observed with Hinode/SOT. Astron. Astrophys. 481, L1. DOI ADS.

Nagashima, K., Sekii, T., Kosovichev, A.G., Zhao, J., Tarbell, T.D.: 2009, Helioseismic Signature of Chromospheric Downflows in Acoustic Travel-Time Measurements From Hinode. Astrophys. J. Lett. 694, L115. DOI ADS

Nagashima, K., Gizon, L., Birch, A., Löptien, B., Couvidat, S., Fleck, B.: 2013, Helioseismic and magnetic imager multi-height doplergrams. In: Shibahashi, H., Lynas-Gray, A.E. (eds.) Progress in Physics of the Sun and Stars: A New Era in Helio- and Asteroseismology, Astron. Soc. Pacific CS-479, 429. ADS

Norton, A.A., Graham, J.P., Ulrich, R.K., Schou, J., Tomczyk, S., Liu, Y., Lites, B.W., López Ariste, A., Bush, R.I., Socas-Navarro, H., Scherrer, P.H.: 2006, Spectral Line Selection for HMI: A Comparison of Fe I $6173 \AA$ and Ni I $6768 \AA$. Solar Phys. 239, 69. DOI. ADS.

Pesnell, W.D., Thompson, B.J., Chamberlin, P.C.: 2012, The Solar Dynamics Observatory (SDO). Solar Phys. 275, 3. DOI ADS

Rajaguru, S.P., Couvidat, S., Sun, X., Hayashi, K., Schunker, H.: 2012, Properties of HighFrequency Wave Power Halos Around Active Regions: An Analysis of Multi-height Data from HMI and AIA Onboard SDO. Solar Phys. 287, 107. DOI ADS

Reale, F.: 2010, Coronal loops: Observations and modeling of confined plasma. Living Rev. Solar Phys. 7(5). DOI ADS

Scherrer, P.H., Schou, J., Bush, R.I., Kosovichev, A.G., Bogart, R.S., Hoeksema, J.T., Liu, Y., Duvall, T.L., Zhao, J., Title, A.M., Schrijver, C.J., Tarbell, T.D., Tomczyk, S.: 2012, The 
Helioseismic and Magnetic Imager (HMI) Investigation for the Solar Dynamics Observatory (SDO). Solar Phys. 275, 207. DOI. ADS

Schou, J., Scherrer, P.H., Bush, R.I., Wachter, R., Couvidat, S., Rabello-Soares, M.C., Bogart, R.S., Hoeksema, J.T., Liu, Y., Duvall, T.L., Akin, D.J., Allard, B.A., Miles, J.W., Rairden, R., Shine, R.A., Tarbell, T.D., Title, A.M., Wolfson, C.J., Elmore, D.F., Norton, A.A., Tomczyk, S.: 2012, Design and Ground Calibration of the Helioseismic and Magnetic Imager (HMI) Instrument on the Solar Dynamics Observatory (SDO). Solar Phys. 275, 229. DOI] ADS

Schou, J., Couvidat, S., Bush, R., Wachter, R., Scherrer, P.H., Rabello-Soares, M.C., et al.: 2014, Post-Launch Calibration and Data Processing for the Helioseismic and Magnetic Imager (HMI) Instrument on the Solar Dynamics Observatory (SDO). Solar Phys. to be submitted.

Stein, R.F.: 2012, Solar Surface Magneto-Convection. Living Rev. Solar Phys. 9(4). DOI. ADS

Stein, R.F., Georgobiani, D., Schafenberger, W., Nordlund, Å., Benson, D.: 2009a, Supergranulation Scale Convection Simulations. In: Stempels, E. (ed.) 15th Cambridge Workshop on Cool Stars, Stellar Systems, and the Sun, AIP, CS-1094, 764. DOI ADS

Stein, R.F., Nordlund, Å., Georgoviani, D., Benson, D., Schaffenberger, W.: 2009b, Supergranulation-Scale Convection Simulations. In: Dikpati, M., Arentoft, T., González Hernández, I., Lindsey, C., Hill, F. (eds.) Solar-Stellar Dynamos as Revealed by Helio- and Asteroseismology: GONG 2008/SOHO 21, Astron. Soc. Pacific CS-416, 421. ADS

Stix, M.: 2002, The sun: an introduction, 2nd edn. Springer. ADS

Straus, T., Fleck, B., Jefferies, S.M., Cauzzi, G., McIntosh, S.W., Reardon, K., Severino, G., Steffen, M.: 2008, The Energy Flux of Internal Gravity Waves in the Lower Solar Atmosphere. Astrophys. J. Lett. 681, L125. DOI ADS

Straus, T., Fleck, B., Jefferies, S.M., McIntosh, S.W., Severino, G., Steffen, M., Tarbell, T.D.: 2009, On the Role of Acoustic-Gravity Waves in the Energetics of the Solar Atmosphere. In: Lites, B., Cheung, M., Magara, T., Mariska, J., Reeves, K. (eds.) The Second Hinode Science Meeting: Beyond Discovery-Toward Understanding, Astron. Soc. Pacific CS-415, 95. ADS

Vernazza, J.E., Avrett, E.H., Loeser, R.: 1981, Structure of the solar chromosphere. III Models of the EUV brightness components of the quiet-sun. Astrophys. J. Suppl. 45, 635. DOI, ADS

Vögler, A., Bruls, J.H.M.J., Schüssler, M.: 2004, Approximations for non-grey radiative transfer in numerical simulations of the solar photosphere. Astron. Astrophys. 421, 741. DOI ADS

Vögler, A., Shelyag, S., Schüssler, M., Cattaneo, F., Emonet, T., Linde, T.: 2005, Simulations of magneto-convection in the solar photosphere. Equations, methods, and results of the MURaM code. Astron. Astrophys. 429, 335. DOI ADS

Wallace, L., Hinkle, K., Livingston, W.: 1998, An atlas of the spectrum of the solar photosphere from 13,500 to 28,000 cm-1 (3570 to 7405 A), N.S.O. Technical Report 98-001, National Solar Observatory. ADS

Wedemeyer, S., Freytag, B., Steffen, M., Ludwig, H.-G., Holweger, H.: 2004, Numerical simulation of the three-dimensional structure and dynamics of the non-magnetic solar chromosphere. Astron. Astrophys. 414, 1121. DOI ADS 